\title{
The Global Magneto-Ionic Medium Survey: Polarimetry of the Southern Sky from 300 to $480 \mathrm{MHz}$
}

\author{
M. Wolleben ${ }^{1,2}$, T. L. Landecker ${ }^{1}$, E. Carretti ${ }^{3,4}$, J. M. Dickey ${ }^{5}$ (D) A. Fletcher ${ }^{6}$, N. M. McClure-Griffiths ${ }^{7}$ (D), D. McConnell ${ }^{3}$, \\ A. J. M. Thomson ${ }^{7}$, A. S. Hill ${ }^{1,8,9}$, B. M. Gaensler ${ }^{10,11}$, J.-L. Han ${ }^{12,13,14}$, M. Haverkorn ${ }^{15}$, J. P. Leahy ${ }^{16}$, W. Reich ${ }^{17}$, and \\ A. R. Taylor ${ }^{18,19}$ \\ ${ }^{1}$ National Research Council Canada, Herzberg Research Centre for Astronomy and Astrophysics, Dominion Radio Astrophysical Observatory, P.O. Box 248, \\ Penticton, British Columbia, V2A 6J9, Canada \\ ${ }^{2}$ Skaha Remote Sensing, 3165 Juniper Drive, Naramata, British Columbia, V0H 1NO, Canada \\ ${ }^{3}$ CSIRO Astronomy \& Space Science, P.O. Box 76, Epping, New South Wales 1710, Australia \\ ${ }^{4}$ INAF-Istituto di Radioastronomia, via P. Gobetti 101, I-40129 Bologna, Italy \\ ${ }^{5}$ University of Tasmania, School of Mathematics and Physics, Hobart, Tasmania 7001, Australia \\ ${ }^{6}$ School of Mathematics, Statistics and Physics, Newcastle University, Newcastle upon Tyne, NE1 7RU, UK \\ ${ }^{7}$ Research School of Astronomy and Astrophysics, Australian National University, Canberra, Australian Capital Territory 2611, Australia \\ ${ }^{8}$ Department of Physics and Astronomy, University of British Columbia, 6224 Agricultural Road, Vancouver, British Columbia, V6T 1Z1, Canada \\ Space Science Institute, Boulder, CO 80301, USA \\ ${ }^{10}$ Sydney Institute for Astronomy, School of Physics, University of Sydney, Sydney, New South Wales 2006, Australia \\ ${ }^{11}$ Dunlap Institute for Astronomy and Astrophysics, University of Toronto, 50 St. George Street, Toronto, M5S 3H4, Canada \\ ${ }^{12}$ National Astronomical Observatories, CAS, Jia-20 DaTun Road, Chaoyang District, Beijing 100101, People's Republic of China \\ ${ }^{13}$ School of Astronomy and Space Sciences, University of the Chinese Academy of Sciences, Beijing 100049, People's Republic of China \\ ${ }^{14}$ CAS Key Laboratory of FAST, NAOC, Chinese Academy of Sciences, Beijing 100101, People's Republic of China \\ ${ }^{15}$ Department of Astrophysics/IMAPP, Radboud University Nijmegen, P.O. Box 9010, NL-6500 GL, Nijmegen, The Netherlands \\ ${ }^{16}$ Jodrell Bank Centre for Astrophysics, Alan Turing Building, School of Physics and Astronomy, University of Manchester, Oxford Road, Manchester, M13 \\ 9PL, UK \\ ${ }^{17}$ Max-Planck-Institut für Radioastronomie, Auf dem Hügel 69, D-53121 Bonn, Germany \\ ${ }^{18}$ Department of Astronomy, University of Cape Town, Rondenbosch 7701, Republic of South Africa \\ ${ }^{19}$ Department of Physics, University of the Western Cape, Republic of South Africa \\ Received 2018 December 20; revised 2019 May 13; accepted 2019 May 17; published 2019 July 2
}

\begin{abstract}
Much data on the Galactic polarized radio emission has been gathered in the last five decades. All-sky surveys have been made, but only in narrow, widely spaced frequency bands-and the data are inadequate for the characterization of Faraday rotation, the main determinant of the appearance of the polarized radio sky at decimeter wavelengths. We describe a survey of polarized radio emission from the southern sky, aiming to characterize the magneto-ionic medium, particularly the strength and configuration of the magnetic field. This work is part of the Global Magneto-Ionic Medium Survey (GMIMS). We have designed and built a feed and receiver covering the band 300-900 MHz for the CSIRO Parkes $64 \mathrm{~m}$ Telescope. We have surveyed the entire sky between decl. $-90^{\circ}$ and $+20^{\circ}$. We present data covering $300-480 \mathrm{MHz}$ with angular resolution $81^{\prime}-45^{\prime}$. The survey intensity scale is absolutely calibrated, based on measurements of resistors at known temperatures and on an assumed flux density and spectral index for Taurus A. Data are presented as brightness temperatures. We have applied Rotation Measure Synthesis to the data to obtain a Faraday depth cube of resolution $5.9 \mathrm{rad} \mathrm{m}^{-2}$, sensitivity of $60 \mathrm{mK}$ of polarized intensity, and angular resolution 1.35. The data presented in this paper are available at the Canadian Astronomy Data Centre.
\end{abstract}

Key words: Galaxy: general - instrumentation: polarimeters - ISM: magnetic fields - radio continuum: ISM surveys - techniques: polarimetric

\section{Introduction}

The first detections of linearly polarized components of the Galactic radio emission were made over fifty years ago (Westerhout et al. 1962; Wielebinski et al. 1962) at frequencies near $400 \mathrm{MHz}$. These discoveries firmly established the synchrotron mechanism as the source of the Galactic nonthermal emission and confirmed the existence of magnetic fields in the Milky Way. The earliest papers commented on the role of Faraday rotation in shaping the appearance of the polarized sky, which was distinctly different from the sky in total intensity. Surveys at other frequencies soon followed (e.g., Berkhuijsen \& Brouw 1963; Mathewson \& Milne 1965; Bingham 1966; Mathewson et al. 1966; Wilkinson 1973; Baker $\&$ Wilkinson 1974). The most comprehensive of these are surveys made during the 1960s with the Dwingeloo $25 \mathrm{~m}$ Telescope, which were reduced in a systematic way and published by Brouw \& Spoelstra (1976). These surveys, at 408, 465, 610, 820, and $1411 \mathrm{MHz}$, cover the entire northern sky, albeit with sparse spatial sampling. Absolutely calibrated and carefully processed, they are considered to be among the best available representations of the polarized sky.

Faraday rotation of the polarized signal from extragalactic sources was detected by Cooper \& Price (1962), and systematic observations over large areas were soon used to generate models of the large-scale Galactic magnetic field (Gardner \& Whiteoak 1963; Seymour 1966; Simard-Normandin \& Kronberg 1979; Brown 2010; Han 2017). Observations at widely spaced frequencies had proved adequate to determine the Rotation Measure (RM) of extragalactic sources, and it was tacitly 
assumed that the same would be true of the Galactic synchrotron emission. On this basis, Spoelstra (1984) calculated the RM of the Galactic emission based on the data at 408, 465, 610, 820, and $1411 \mathrm{MHz}$ of Brouw \& Spoelstra (1976).

In contrast, the complexity of Faraday effects in a medium where emission and Faraday rotation are mixed had already been demonstrated by Burn (1966). Burn showed that, when synchrotron emission and Faraday rotation are present in the same volume, as must often be the case in the Galaxy, "RM" is not a meaningful concept and measurements in many closely spaced frequency channels are required in order to fully characterize Faraday rotation. However, the technology of the 1960s was inadequate to collect or process signals with the necessary bandwidth and frequency resolution.

All-sky surveys at single frequencies (e.g., Brouw \& Spoelstra 1976; Wolleben et al. 2006; Testori et al. 2008) and aperture-synthesis surveys in the Galactic plane (Haverkorn et al.

2006; Landecker et al. 2010) have provided two-dimensional mapping of the Galactic magnetic field. In combination with other tracers, such as Faraday rotation toward point sources (Han et al. 2006; Brown et al. 2007; Taylor et al. 2009), they have contributed to reconstructions of the three-dimensional structure of the Galactic magnetic field (Sun et al. 2008; Jansson \& Farrar 2012).

Today, advances in antennas, receivers, and digital signal processing have made polarimetry possible over wide bands with many frequency channels. The technique of RM Synthesis (Brentjens \& de Bruyn 2005), drawing on the concepts of Burn (1966), has been developed and applied to aperture synthesis observations (de Bruyn \& Brentjens 2005). Our Global Magneto-Ionic Medium Survey (GMIMS) exploits these opportunities. With GMIMS, we set out to map the polarized radio emission from the entire sky, in the northern and southern hemispheres, using large single-antenna radio telescopes, covering the entire frequency range $300-1800 \mathrm{MHz}$ (Wolleben et al. 2009), and to use RM Synthesis to analyze the data. The frequency band has been notionally divided in three, $300-800 \mathrm{MHz}, \quad 800-1300 \mathrm{MHz}$, and $1300-1800 \mathrm{MHz}$, to define the Low, Mid, and High GMIMS bands. Because any one telescope can only see just over half the sky, we obviously required north and south surveys in each of the three bands. We developed the techniques for GMIMS with a survey of the High GMIMS band made with the John A. Galt Telescope at the Dominion Radio Astrophysical Observatory (diameter $26 \mathrm{~m}$ ), and we have now completed a spectropolarimetric survey of the northern sky covering $1280-1750 \mathrm{MHz}$ using that telescope. Scientific results from this survey have been published by Wolleben et al. (2010), Sun et al. (2015a), and Hill et al. (2017), and the survey itself will be described in a forthcoming paper (M. Wolleben et al 2019, in preparation). We have also used the CSIRO Parkes $64 \mathrm{~m}$ Telescope to make two surveys of the southern sky: one in the High band and one in the Low. In this paper, we describe the Parkes survey in the Low Band.

The motivation for the GMIMS project is our conviction that the magnetic field is an important energy-carrying constituent of the interstellar medium (ISM). Ferrière (2001) envisages the ISM with three principal constituents: gas (in cold $\left[\sim 10^{2} \mathrm{~K}\right]$, warm $\left[\sim 10^{4} \mathrm{~K}\right]$, and hot $\left[\sim 10^{6} \mathrm{~K}\right]$ thermally stable "phases"), magnetic fields, and cosmic rays. On large scales, the energy densities of these constituents are in approximate equipartition. Nevertheless, there are substantial local and scale-dependent deviations from equilibrium (Wolfire et al. 2003; Joung et al. 2009; Heiles \& Haverkorn 2012), and we cannot understand any one constituent of the ISM in isolation. For example, the presence of the magnetic field and other nonthermal pressure components can fundamentally change the character of the ISM from one dominated by hot gas with embedded warm and cold clouds (McKee \& Ostriker 1977; Li et al. 2015) to one dominated by warm gas with embedded cold clouds and hot supernova remnants (Slavin \& Cox 1993; de Avillez \& Breitschwerdt 2004, 2005; Gressel et al. 2008). Overall, thermal and nonthermal pressures provide the vertical support that keeps the ISM in hydrostatic equilibrium (Boulares \& Cox 1990; Piontek \& Ostriker 2007; Gressel et al. 2008; Ostriker et al. 2010; Hill et al. 2012), and the multiphase turbulent cascade in the ISM on all scales is partly controlled by magnetism (Armstrong et al. 1995; Minter \& Spangler 1996; Chepurnov \& Lazarian 2010).

Observational studies of the three-dimensional distribution of the ISM gas are in a far more advanced state than those of the magnetic field and cosmic rays. There are now all-sky, kinematically resolved surveys of $\mathrm{H}$ I emission with sub-degree angular resolution (Kalberla et al. 2005; McClure-Griffiths et al. 2009; Kerp et al. 2011; Ben Bekhti et al. 2016), as well as arcminute-resolution surveys of smaller areas, both completed (Taylor et al. 2003; McClure-Griffiths et al. 2005, 2012; Peek et al. 2011, 2018) and planned (Dickey et al. 2013). There is also an all-sky, kinematically resolved survey of $\mathrm{H} \alpha$ emission from the warm ionized ISM (Haffner et al. 2003, 2010). ${ }^{20}$ Through the velocity dimension, these surveys provide information on the distribution, structure, and kinematics of neutral and ionized gas in the Milky Way. With GMIMS, we aim to provide an all-sky counterpart to these surveys by mapping polarized emission from the magneto-ionized ISM, with information on the third dimension provided by Faraday depth. While Faraday depth is not a direct proxy for distance, it can provide information on the three-dimensional structure of the magnetic field-information that is not accessible in any other way.

Dust intermingles with the ISM gas. It is small in mass fraction, amounting to $\sim 1 \%$ of the ISM, but crucial for interstellar chemistry and star formation. Dust grains are aligned by magnetic fields, and dust observations play a major role in studies of the Galactic magnetic field (e.g., Planck Collaboration et al. 2016). Dust polarization traces the magnetic field in the plane of the sky, while Faraday effects trace the field in the line of sight: the two kinds of observations are complementary.

In this paper, we describe a polarization survey of the southern sky using the Parkes $64 \mathrm{~m}$ Telescope. The initial goal was to map the sky from 300 to $900 \mathrm{MHz}$, but strong radiofrequency interference (RFI) prevented this. We present data between 300 and $480 \mathrm{MHz}$ covering $2.68 \pi$ steradians, $67.1 \%$ of the whole sky. Section 2 describes the feed and receiver designed and built for this survey. Survey observations are described in Section 3. In Section 4, we describe the methods used to calibrate the survey in terms of absolute standards of noise. Data processing is the subject of Section 5. Section 6 probes the quality of the survey data through comparisons with existing data. Results are presented in Section 7 and discussed in Section 8.

\footnotetext{
${ }^{20}$ http://www.astro.wisc.edu/wham-site/wham-sky-survey
} 


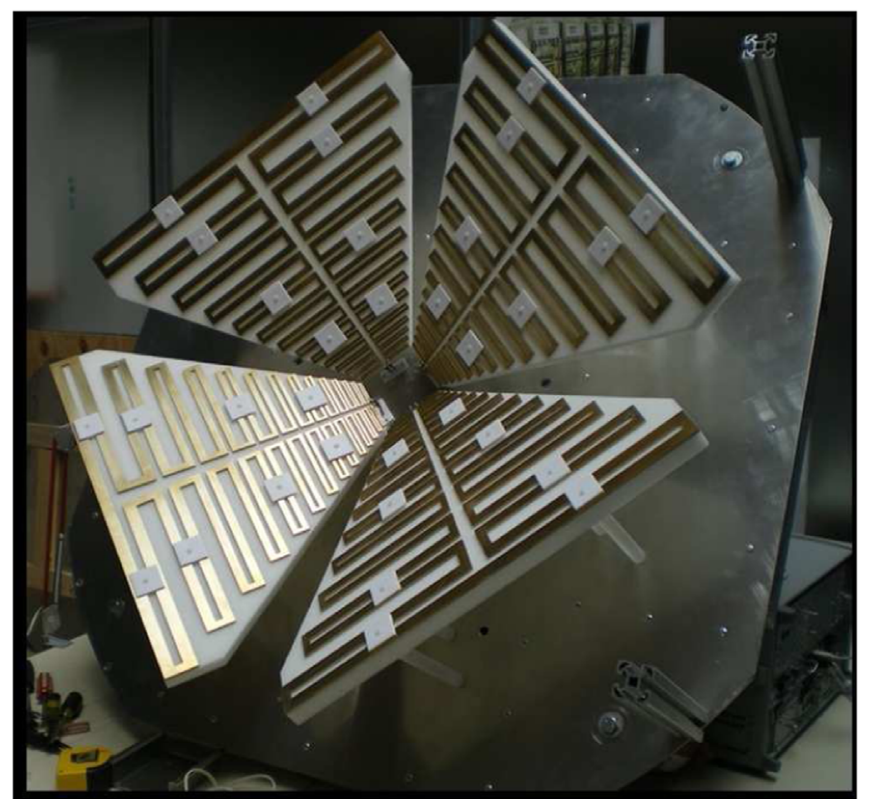

Figure 1. The feed, designed and built for this survey. Four petals, each a logperiodic array of folded dipoles, are supported above a ground plane of dimensions $1.2 \times 1.2 \mathrm{~m}$.

\section{Feed and Receiver}

The target band, $300-900 \mathrm{MHz}$, was chosen as a balance between a need to reach low frequencies to achieve good resolution in Faraday depth, limitations imposed by RFI at the Parkes observatory, the $1 \mathrm{GHz}$ input bandwidth of the available digital signal processor, and the feasibility of building an appropriate feed to collect the signals. Neither feed nor receiver was available for the telescope covering our chosen band; this section of the paper describes their design and implementation.

\subsection{Feed Design}

The specifications for the feed called for constant illumination of the $64 \mathrm{~m}$ reflector over the band $300-900 \mathrm{MHz}$, and the delivery of left-hand and right-hand circular polarization (which we denote by $L$ and $R$ ) to the receiver and the digital signal processor. The Parkes Telescope has a diameter, $D$, of $64 \mathrm{~m}$ and a focal length, $f$, of $26 \mathrm{~m}$. The value of $f / D$ is 0.41 , and the opening angle of the reflector as seen from the focus is $126^{\circ}$.

Our design is based on the Eleven Feed, an invention of Kildal (2005), which has been shown to be able to meet a variety of wideband needs (Olsson et al. 2006; Yang et al. 2011). The basic element of the Eleven Feed is a pair of parallel half-wave dipoles above a ground plane: such a feed can easily be built to provide a circularly symmetric illumination of a reflector of opening angle $\sim 120^{\circ}$. In the Eleven Feed, each dipole is expanded to become an approximately log-periodic array of folded dipoles fed by a twin-wire transmission line. We refer to this structure as a petal. Our feed, shown in Figure 1, consists of four petals, each connected to the receiver at the short-dipole end. For any operating wavelength, $\lambda$, the dipoles of length $\sim 0.5 \lambda$ are resonant, and the feed is effectively a pair of parallel half-wave dipoles separated by about $0.45 \lambda$ and close to $0.15 \lambda$ above the ground plane (Olsson et al. 2006). We refined this basic design using the Computer Simulation Technology (CST) package (CST 2014). Starting from an array

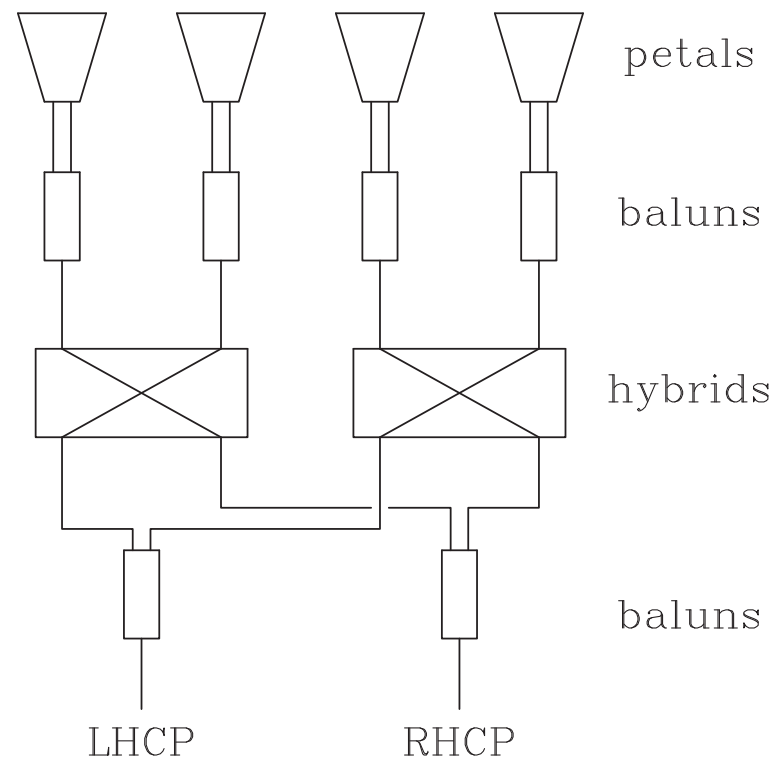

Figure 2. Diagram of the phasing network. The petals are the radiating elements, connected through baluns to the $90^{\circ}$ hybrids, which combine signals in quadrature. Two hands of circular polarization emerge from the lower layer of baluns, which are used as $180^{\circ}$ hybrids.

of 13 dipoles and a replication factor of 1.15, we used the optimization routines within CST to improve the performance. The challenge in designing such a feed is to maintain constant beamwidth and gain across the operating band, while at the same time achieving an acceptable impedance match. Using its internal genetic-algorithm optimizer, we allowed CST to vary dipole length, the width of the dipole arms, and the angle of the petal above the ground plane (the latter parameter should be constant in a truly frequency-independent feed). Each iteration was evaluated by considering beamwidth at the $-10 \mathrm{~dB}$ points (desirable width $\sim 120^{\circ}$ ), the closely related forward gain (desirable value $10 \mathrm{~dB}$ ), and input return loss (desirable value $10 \mathrm{~dB}$ ). Return loss was based on a characteristic impedance of $200 \mathrm{ohms}$ for the balanced line. A petal thickness of $3.2 \mathrm{~mm}$ was assumed throughout the simulation (a departure from log periodicity, which is more significant at the high end of the band). In the final design, the shortest and longest dipoles have respective lengths of 9 and $61 \mathrm{~cm}$, corresponding to respective frequencies of 1665 and $245 \mathrm{MHz}$.

Each petal was connected by a twin-wire line to a balun. A Marchand balun was used, and impedance transformationfrom $200 \mathrm{ohms}$ on the balanced side to $50 \mathrm{ohms}$ on the unbalanced side-was built into it. The design is derived from the ideas presented in Puglia (2002). A return loss $>10 \mathrm{~dB}$ was achieved across the band $300-1300 \mathrm{MHz}$; phase balance was within $5^{\circ}$.

Two sets of petals were placed orthogonally to accept two linear polarizations. The petals were connected to a network that excited them at phases $0^{\circ}, 90^{\circ}, 180^{\circ}$, and $270^{\circ}$, so that $L$ and $R$ could be generated: the phasing network is illustrated in Figure 2. It employed TEM-line hybrids to generate $90^{\circ}$ phase shifts and balun circuits to generate $180^{\circ}$ phase shifts. The baluns were similar to those connected to the petals, but transformed between $50 \mathrm{ohms}$ unbalanced and two $50 \mathrm{ohm}$ unbalanced outputs in antiphase-the equivalent of $100 \mathrm{ohms}$ balanced. They were fitted with connectors for simple interconnection of the phasing elements. 


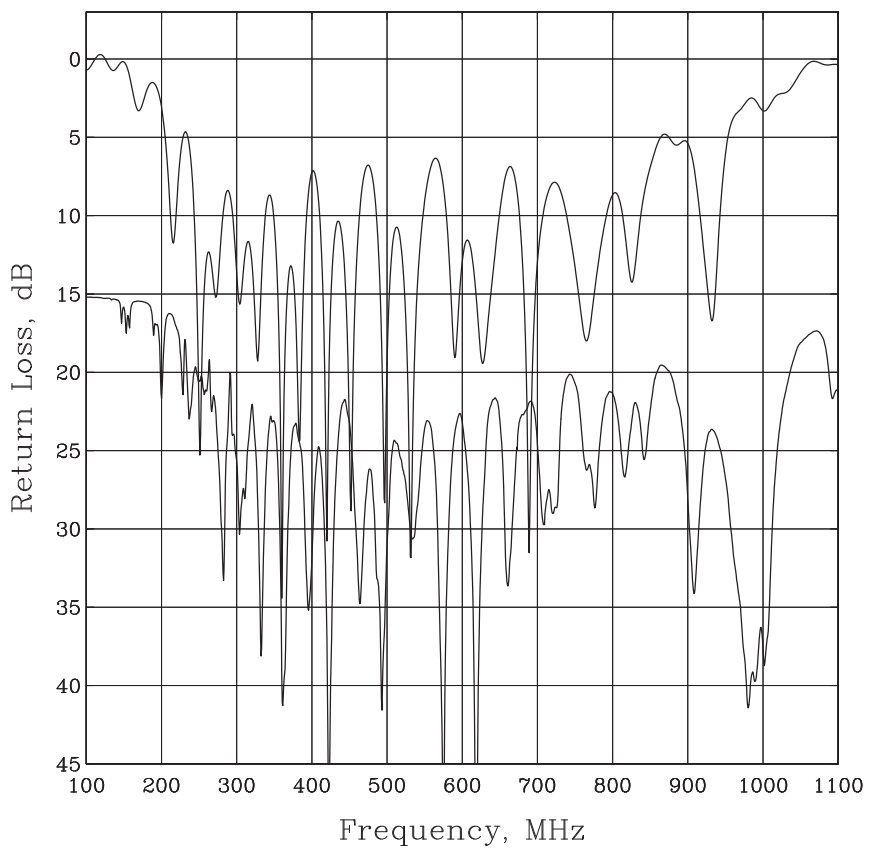

Figure 3. Calculated return loss of one polarization of the feed (top curve) Measured return loss of one petal (bottom curve-displaced by $15 \mathrm{~dB}$ for clarity).

\subsection{Feed Fabrication and Construction}

The petals were fabricated by water-jet cutting from a $1 / 8$ inch brass sheet. The thickness was chosen because thick radiating structures have lower loss than thin ones. The material was chosen to allow the baluns to be soldered to the radiating elements. Each petal was glued to low-loss dielectric foam, ${ }^{21}$ and the assembly was supported above the ground plane on eight polystyrene rods $19 \mathrm{~mm}$ in diameter. The twin-wire line between the petals and the baluns was made of stranded copper wire to maintain the flexibility of this important connection (the feed is subject to considerable vibration on the telescope). The baluns were fabricated as printed circuits on standard FR4 circuit board. The TEM hybrids were commercial devices. ${ }^{22}$

\subsection{Feed Performance}

Figure 3 presents results for the return loss of a single petal correctly placed above the ground plane. Measurements were made through the balun. The other three petals were terminated with matched resistors. Simulation suggested that the return loss of the feed as a whole closely resembled that of a single petal, but the return loss of the assembled feed was impossible to measure directly. It is clear that the manufactured feed matched the simulation well, which both gave confidence in our ability to simulate the feed and demonstrated that the balun is essentially transparent. However, we see that our design did not reach its target; the worst-case return loss was $\sim 5 \mathrm{~dB}$, and in narrow frequency ranges about $30 \%$ of the power in the incoming signal was reflected. In the band for which results are presented in this paper, $300-480 \mathrm{MHz}$, minimum return loss was $7 \mathrm{~dB}$. Averaged over that band, the net power transfer efficiency to a perfectly matched termination would have been $93.4 \%$, but we note that poor matching of the feed was

\footnotetext{
21 Cuming Microwave C-STOCK RH5.

22 R\&D Microwaves model HD-A01.
}

confined to narrow and separated frequency ranges. The receiver components, especially the Low-Noise Amplifier (LNA), were not perfectly matched, so power transfer may have been slightly higher or lower than this figure. No correction was necessary for feed mismatch, because the primary calibrator (Taurus A; see Section 4) was, of course, observed through the same feed.

Measuring the feed radiation characteristics was more challenging: the feed was big and heavy, and we did not have access to a sophisticated antenna range where accurate measurements would have been possible. We simply set up the feed outdoors, about one meter above the ground, and made measurements on a circle of radius $10 \mathrm{~m}$ centered on the feed. We could measure E- and H-plane patterns approximately, but it was not possible to measure cross-polarization performance. Figure 4 shows the resulting radiation pattern measurements, along with patterns calculated in the simulations. At all frequencies below $700 \mathrm{MHz}$, the feed radiation patterns were symmetrical (although this is not shown in Figure 4), and the patterns were approximately circular. We judged that radiation past the edge of the reflector (spillover) would be at an acceptably low level.

Measured feed performance at 700 and $800 \mathrm{MHz}$ departed from simulations. This may be connected to an abrupt change in measured beamwidth of the telescope at $\sim 800 \mathrm{MHz}-$ see Section 4 and Figure 7.

\subsection{Losses in the Feed and Phasing Network}

Losses in the feed and the phasing network affect the calibration of the survey. The insertion losses of these components were measured directly, wherever possible, using a network analyzer. Where direct measurement was not possible, measurements of return loss were used (also made with a network analyzer). When the output of a device is short circuited, a signal injected into its input will pass through the device, be reflected, and return to the input. The signal passes through the device twice, and the insertion loss is then half the measured return loss.

To measure the loss in the feed, a signal was transmitted into it and reflected back into its input. To achieve total reflection, the feed was completely surrounded by a metal box $1.2 \times 1.2 \times 1.04 \mathrm{~m}$. This box contacted the ground plane of the feed (size $1.2 \times 1.2 \mathrm{~m}$ ). Two measurements were made, one with a box depth of $1.04 \mathrm{~m}$ and one with a depth of $0.9 \mathrm{~m}$; the results were identical within the errors. This technique is essentially the Wheeler cap method (Wheeler 1959).

The $200 \mathrm{ohm}$ baluns were soldered in place and could not be measured separately. It was assumed that they have the same loss as the $50 \mathrm{ohm}$ baluns (a reasonable assumption because the loss was mostly in the FR4 circuit board).

Total losses in the feed and phasing network range from $0.80 \mathrm{~dB}$ at $300 \mathrm{MHz}$ to $1.1 \mathrm{~dB}$ at $500 \mathrm{MHz}$ and $1.26 \mathrm{~dB}$ at $900 \mathrm{MHz}$. Loss in the feed itself was calculated by subtracting balun loss from the results of the measurement described above. The estimated accuracy of the losses determined in this series of measurements is $\pm 0.25 \mathrm{~dB}$ or $\pm 5 \%$. Some error is expected because currents in a device with its output shortcircuited are not identical with currents under normal use, but the technique certainly gives credible upper limits to loss. Measured losses are summarized in Figure 5.

We note that the Eleven Feed has fairly high loss. The signal at any frequency in the band travels from the dipole actively 


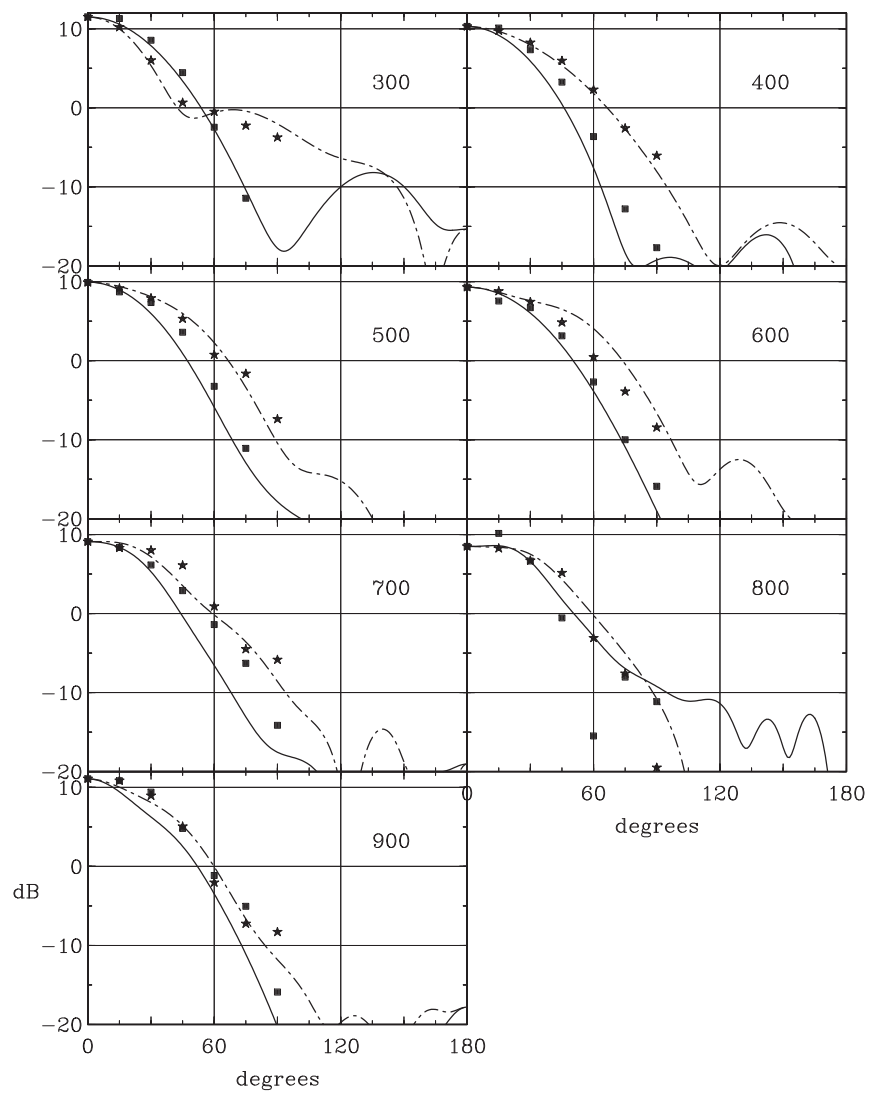

Figure 4. Radiation patterns of the feed. The calculated patterns of the E-plane are shown as solid lines, and the H-plane as dashed-dotted lines. The measured patterns of the E-plane are shown as squares, and the H-plane as stars. Frequency $(\mathrm{MHz})$ is shown in each panel.

receiving that frequency through all the shorter dipoles before reaching the balun. In our implementation, the length of that path is about one wavelength at any given frequency in the band.

\subsection{Receiver}

The receiver was very straightforward. It had no frequency conversion, but simply amplified the received signals to a level where they could be transmitted via coaxial cable from the telescope focus cabin to the receiver room (a distance of about $100 \mathrm{~m}$ ). The LNAs employed were commercial devices that gave a very flat passband but not exceptionally low noise temperature. The excess noise, $T_{e}$, of the LNAs was $\sim 70 \mathrm{~K}$. System temperature varied from $115 \mathrm{~K}$ at $300 \mathrm{MHz}$ to $145 \mathrm{~K}$ at $900 \mathrm{MHz}$ without the feed. Losses of $0.8-1.2 \mathrm{~dB}$ in the feed and phasing network (see Figure 5) added $55-85 \mathrm{~K}$ to the system noise.

Bandpass filters following the LNAs defined two bands, 290-470 MHz and 660-870 MHz. Outside these two bands, the level of RFI was so high that radio astronomy measurements were not possible. Received signals were analyzed with a digital spectropolarimeter of input bandwidth $1 \mathrm{GHz}$, able to form all products $L L^{*}, R R^{*}, R L^{*}$, and $L R^{*}$ from the input $L$ and $R$ signals, allowing the derivation of all four Stokes parameters in 2048 frequency channels. The Stokes parameters were calculated as $I=0.5\left(R R^{*}+L L^{*}\right), Q=L R^{*}$, and $U=R L^{*}$. A noise source in a temperature-controlled enclosure was coupled equally into both $L$ and $R$ paths with equal phase (after the feed and phasing network), providing a linearly polarized calibration signal.

\section{Survey Observations}

All survey observations used the technique developed for the $S$-band Parkes All-Sky Survey with the same telescope (Carretti et al. 2019). The telescope was scanned rapidly, at $15^{\circ}$ per minute, in azimuth, $A$, at the elevation of the South Celestial Pole, $33^{\circ}$, with the feed fixed in the telescope frame. Ground radiation is essentially constant as a function of azimuth (the terrain around the telescope is quite flat). Fixed contributions to the observed $Q$ and $U$, particularly ground radiation and instrumental polarization, remained constant throughout a scan. The polarized signal from the sky, however, was modulated by the changing parallactic angle, producing a sinusoidal variation along the scan. The two contributions could therefore be separated in data processing, and the ground and instrumental contributions removed, preserving the sky signal on all scales. The key to successful application of this technique is long azimuth scans, to produce the greatest possible range of parallactic angle.

Scans were either "east" scans $\left(A<180^{\circ}\right)$ or "west" scans $\left(A>180^{\circ}\right)$, where $A=180^{\circ}$ is south. We observed either the setting sky or the rising sky: this strategy produced scans that crossed one another at large angles, enhancing the benefits of basketweaving (see Section 5.7). West scans ran from $A=180^{\circ}$ to $A=290^{\circ}$. Short east scans ran from $A=180^{\circ}$ to $A=30^{\circ}$, and long east scans ran from $A=180^{\circ}$ through $0^{\circ}$ to $A=290^{\circ}$. This scanning potentially gave complete coverage of the sky from decl. $\delta=+24^{\circ}$ to $\delta=-90^{\circ}$. However, the master equatorial, to which telescope movement is locked, could not be driven further south than $\delta=-87^{\circ}$. West scans covered decl. $-87^{\circ}$ to $0^{\circ}$ and east scans covered decl. $-87^{\circ}$ to $+20^{\circ}$. Data for the small area around the South Celestial Pole were acquired during ramp-up time for scans nominally starting at decl. $=-87^{\circ}$. Start times of scans were not arbitrary, but were spaced by 10.5 , chosen to ensure complete (Nyquist) sampling of the sky at $900 \mathrm{MHz}$, where the half-power beamwidth is $\sim 25^{\prime}$.

Observations were made exclusively at night to avoid contamination from solar emission entering through sidelobes. Observations were scheduled in blocks of 12-20 nights, spaced throughout the year so that all R.A. could be covered. Altogether, about $2000 \mathrm{hr}$ of observing time were allocated, on the dates listed in Table 1.

\section{Absolute Calibration}

We have made it a priority that all the surveys that comprise the GMIMS data set should be absolutely calibrated. With their wide frequency coverage, these surveys extend far beyond the traditional radio astronomy frequency allocations, and few or no absolutely calibrated data are available that we can tie our surveys to. While the existing absolute calibrations are technically very strong, they are old, and it is worthwhile to corroborate them with modern measurements.

In the calibration of a polarization survey, there are two separate challenges: calibration of the brightness temperature scale and calibration of polarization angle. We were able to calibrate the brightness temperature scale with simple techniques, but we were not able to calibrate polarization angle and eventually had to rely on published data for the latter (see 


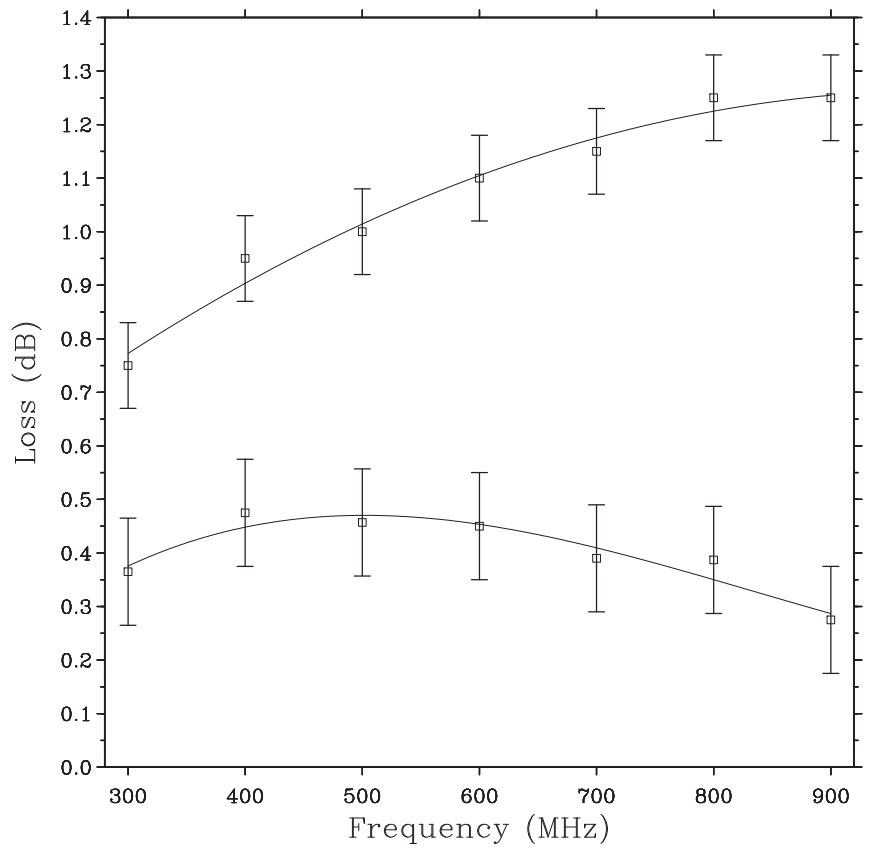

Figure 5. Measured loss in the feed and circuits that precede the point where the calibration noise signal is injected. The upper curve displays the total measured loss, and the lower curve the loss in the feed.

Section 6.2). There are strong sources with high fractional polarization (e.g., 3C 286 and 3C 138) whose characteristics are very well-known; they are useful calibrators of polarization angle for telescopes of high angular resolution at frequencies above $\sim 1 \mathrm{GHz}$. At the frequencies that we used in this project, $300-900 \mathrm{MHz}$, there are no strong polarized sources in the southern sky-certainly none strong enough to yield a significant signal in the beam, $0.4-1.3$. In this frequency range, it is possible to calibrate using pulsars (e.g., Liao et al. 2016), but that option was not open to us.

The goal of absolute calibration is to measure the sky brightness temperature in Kelvins on a scale tied to absolute standards of thermal noise-resistors at known temperatures. To achieve this, we need to measure or calculate some telescope characteristics.

We define the radio telescope by its power response $f(\theta, \phi)$, where $\theta, \phi$ are spherical coordinates and $f(0,0)=1$. If the telescope is immersed in a temperature distribution $T(\theta, \phi)$, the available power at its terminals is equivalent to the antenna temperature,

$$
T_{A}=\frac{1}{\Omega} \int_{4 \pi} T(\theta, \phi) f(\theta, \phi) d \omega .
$$

Here, $d \omega$ is the element of solid angle, and $\Omega$ is the antenna solid angle defined by

$$
\Omega=\int_{4 \pi} f(\theta, \phi) d \omega
$$

A large reflector antenna like the Parkes telescope directs feed radiation into a main beam and sidelobes. Choosing a convenient boundary between them, we can separate $\Omega$ into main-beam solid angle, $\Omega_{B}$, and sidelobe solid angle, $\Omega_{S}$, and we can similarly separate $T_{A}$ into main beam and sidelobe contributions, $T_{A B}$ and $T_{A S}$. The closest approximation to the true brightness distribution over the main beam that the finite aperture of the telescope allows us to measure is

$$
T_{B}=\frac{\Omega}{\Omega_{B}} T_{A B}=\frac{\Omega}{\Omega_{B}}\left(T_{A}-T_{A S}\right) .
$$

Derivation of $T_{B}$ therefore requires correction of observations for the contribution from sidelobes, which in turn demands full knowledge of the sidelobes. This is a difficult assignment when mapping the total-intensity sky with a large telescope. The problem is circumvented for the polarized sky because $Q$ and $U$ take on positive and negative values and tend to average to a very small number over large areas. Exceptions arise for spillover lobes interacting with the ground: spillover lobes, far from the telescope axis, have very non-ideal polarization properties, and can convert unpolarized ground emission to apparently polarized signal. Our mapping technique overcame this, and ground signal was effectively removed from the signal stream (see Section 5.7).

The antenna solid angle, $\Omega$, is a measure of the ability of the telescope to concentrate radiation from the feed into a beam, or conversely, the ability of the telescope to collect radiation from a small area of the sky. This ability can (equivalently) be expressed in terms of an effective area of the telescope, $A_{e}$. Contrasting the antenna with an isotropic radiator (a theoretical construct), the gain of the antenna over the isotropic radiator is

$$
G=\frac{4 \pi}{\Omega}=\frac{4 \pi A_{e}}{\lambda^{2}} .
$$

The aperture efficiency, $\eta_{A}$ describes the utilization of the telescope aperture: it is the effective area, $A_{e}$, divided by the physical area, $A_{p}$

$$
\eta_{A}=\frac{A_{e}}{A_{p}} .
$$

The beam efficiency is

$$
\eta_{B}=\frac{\Omega_{B}}{\Omega}
$$

Several approaches to absolute calibration are possible. For instance, $\Omega$ can be measured by measuring the power response, $f(\theta, \phi)$ for all directions $(\theta, \phi)$, but this is impractical for the Parkes telescope. One can also calculate $\Omega$ from the known dimensions of the telescope, but $\mathrm{Du}$ et al. (2016) show that simulation software cannot yet achieve the desired precision. Finally, the increase in antenna temperature, $\Delta T_{A}$, when a radio source of known flux density $S$ is centered in the main beam can yield $\Omega$ through the relationship

$$
\Delta T_{A}=\frac{S \lambda^{2}}{2 k \Omega} .
$$

Here, $k$ is Boltzmann's constant and flux density, $S$, is measured in Jy. We chose this method because there are strong calibration sources that have well-established flux densities, accurate to a few percent.

There are no strong, compact sources in the southern sky with precisely known flux densities and spectral indices, so we chose one from the northern sky. The strong northern sources with accurately known flux densities that are within the decl. range of the Parkes Telescope are Virgo A (decl. $\sim 12^{\circ} .4$ ) and Taurus A (decl. $22^{\circ} .0$ ). We chose Taurus A because it is the more powerful of the two, with the better determined absolute 
Table 1

Survey Parameters

\begin{tabular}{|c|c|}
\hline Beam FWHM & $88^{\prime}$ at $300 \mathrm{MHz}, 45^{\prime}$ at $480 \mathrm{MHz}$ \\
\hline Channel bandwidth & $0.5 \mathrm{MHz}$ \\
\hline Aperture efficiency & $\sim 27 \%$ \\
\hline Beam efficiency & $55 \%$ at $300 \mathrm{MHz}, 43 \%$ at $480 \mathrm{MHz}$ \\
\hline Integration time & $0.25 \mathrm{~s}$ \\
\hline Decl. range & $-90^{\circ}$ to $+20^{\circ}$ \\
\hline System temperature & $250 \mathrm{~K}$ \\
\hline \multirow[t]{10}{*}{ Observing dates } & 2009 Sep 7-2009 Sep 21 \\
\hline & 2009 Nov 30-2009 Dec 9 \\
\hline & 2010 Feb 23-2010 Mar 9 \\
\hline & 2010 Jun $25-2010$ Jul 8 \\
\hline & 2010 Aug 26-2010 Sep 10 \\
\hline & 2010 Nov 10-2010 Nov 24 \\
\hline & 2011 Feb 9-2011 Feb 23 \\
\hline & 2011 Oct 20-2011 Nov 10 \\
\hline & 2012 Feb 8-2012 Feb 29 \\
\hline & 2012 Jun 8-2012 Jul 2 \\
\hline Resolution in Faraday depth & $5.9 \mathrm{rad} \mathrm{m}^{-2}$ \\
\hline Largest scale in Faraday depth & $8.6 \mathrm{rad} \mathrm{m}^{-2}$ \\
\hline Maximum observable Faraday depth & $1700 \mathrm{rad} \mathrm{m}^{-2}$ \\
\hline
\end{tabular}

spectrum. We discuss the value that we have adopted for the flux density of Taurus A in Section 4.2.

\subsection{Measuring the Calibration Signal}

The equivalent temperature in Kelvins of the injected noise signal was measured in 2010 June, 2011 February, and 2012 February. Measurements were made separately in the $L$ and $R$ channels, relative to resistive terminations at ambient temperature and in a liquid-nitrogen bath. These measurements were straightforward. Calibration signal amplitude was $24-47 \mathrm{~K}$ across the frequency band. The error, estimated from differences in the three measurements, was $\sim 0.7 \mathrm{~K}$. Feed mismatch, discussed in Section 2.3, had no effect on the calibration signal nor on the accuracy of our measurements of it. The calibration noise signal always worked into a wellmatched termination, and only a small fraction of the noise power was coupled into the receiver through directional couplers.

\subsection{Measuring Aperture Efficiency}

At least once in every observing session (see Table 1), a raster scan was made of Taurus $A$, covering an area $5^{\circ} \times 5^{\circ}$. The beamshapes deduced from these observations were very closely Gaussian in profile. We averaged ten channels between 360.75 and $370.75 \mathrm{MHz}$ (near the center of our survey band). We removed a twisted-plane baseline from the map (justified because Taurus $\mathrm{A}$ is near the Galactic plane). In Figure 6, we show cross sections through the beam in R.A. and decl. and Gaussian fits to those profiles. Although the Gaussians were fitted to the individual cross sections, they are consistent in width within the errors. The $L L$ and $R R$ beams were coincident.

The individual raster scans of Taurus A were processed as follows. A two-dimensional Gaussian over a twisted-plane base was fitted to the map and the amplitude and half-power widths of the Gaussian were tabulated. Widths were corrected for the finite extent of Taurus A. Figure 7 shows the half-width of the Gaussian, averaged over twelve scans, and the polynomial fitted to the average; the telescope beamwidth was taken from this fitted curve. Each of the twelve scans was affected by RFI.
RFI-affected channels were flagged and mostly did not participate in the average. Nevertheless, the impact of RFI can be seen clearly in Figure 7 from $400 \mathrm{MHz}$ to the top of the band. $^{23}$

The adopted flux density of Taurus A is $1450 \mathrm{Jy}$ at $300 \mathrm{MHz}$ with a spectral index of -0.299 . These values were taken from the VLSS Bright Source Spectral Calculator (Ida10g.alliance. unm.edu/calspec/calspec.html) in 2008. The flux density value was consistent with the spectrum for Taurus A established by Baars et al. (1977), which covered frequencies down to $1000 \mathrm{MHz}$, but the website entry also took into account more recent data at lower frequencies, and therefore seemed to us to account for a possible decline in the flux density of the source. However, the website flux density has subsequently been revised to $1407 \mathrm{Jy}$, citing errors in the survey paper (Helmboldt et al. 2008). This notwithstanding, we believe $1450 \mathrm{Jy}$ is a good value for the following reasons. Taking numbers from Baars et al. (1977) and extrapolating below $1000 \mathrm{MHz}$ to $300 \mathrm{MHz}$ gives a flux density of $1494 \mathrm{Jy}$ for epoch 1980. As might be expected for a supernova remnant, the flux density of Taurus A is apparently declining: Aller \& Reynolds (1985) measured a decline of $0.167 \%$ per year at $8.0 \mathrm{GHz}$, and Vinyaikin (2007) find a similar rate of decline at 151.5 and $927 \mathrm{MHz}$. Applying this rate of decline to the Baars et al. (1977) value over the $30 \mathrm{yr}$ from 1980 to 2010 gives a flux density of $1419 \mathrm{Jy}$ at $300 \mathrm{MHz}$. Our adopted value of $1450 \mathrm{Jy}$ is within $3 \%$ of all of these numbers. We accept a slightly higher number, 5\%, as the probable error in our adopted flux density; this covers the range of values permitted by the parameters for Taurus A given by Baars et al. (1977). Our adopted spectral index, $-0.299 \pm$ 0.009 , is the spectral index derived by Baars et al. (1977). We note that many of the measurements used by Baars et al. (1977) were made with horn antennas and dipole arrays for which the conversion from Kelvins of antenna temperature to Janskys can be accurately calculated. These are therefore absolute measurements, and we consider our survey to be absolutely calibrated.

The amplitudes of the fitted Gaussians were converted to temperature units by comparison with the calibration noise source. This gave the antenna temperature, $T_{A}(\mathrm{TauA})$, as a function of frequency. Given the adopted flux density and spectral index, we were able to calculate the aperture efficiency, $\eta_{A}$, of the telescope; the result is shown in Figure 8. Aperture efficiency measured in this way includes the loss of the feed and phasing network (see Section 2.4 and Figure 5). A second curve in Figure 8 shows aperture efficiency corrected for feed loss. It is very unlikely that $\eta_{A}$ has fine frequency structure. We believe that the apparent depression in $\eta_{A}$ below $320 \mathrm{MHz}$ and the rapid fluctuations of $\eta_{A}$ from 380 to $480 \mathrm{MHz}$ arise from vestiges of RFI in the data. The best approximation to $\eta_{A}$ is a constant value of $0.32 \pm 0.01$ across the band after correction for loss in the feed.

We expected a value of $\eta_{A} \approx 45 \%$ for the Parkes Telescope with a feed of this type. From the radiation patterns of the feed (Figure 4), we calculated illumination efficiency, $\eta_{I}$, and spillover efficiency, $\eta_{S}$; from telescope dimensions, we calculated blockage efficiency, $\eta_{B}$, taking into account both plane-wave and spherical-wave blockage (see Du et al. (2016)

\footnotetext{
23 The rapid fall in deduced beamwidth above $\sim 800 \mathrm{MHz}$ is not completely understood. It may be related to departures of measured feed performance from simulations near that frequency discussed in Section 2.3, but it may also be a result of remanent RFI in the Taurus A data below or above $800 \mathrm{MHz}$. We have not concerned ourselves with this effect, as this paper does not deal with data in that frequency range.
} 
for an explanation of these terms). At our low frequencies, other factors (surface errors and surface transparency) are negligible for the Parkes telescope. We derived $\eta_{I} \approx 0.55$, $\eta_{S} \approx 0.85$, and $\eta_{B} \approx 0.9$, and their product gave a predicted $\eta_{A} \approx 0.42$. What could have reduced this value to 0.32 ? Our hypothesis is that the feed was not quite at the focus of the telescope, but we presently have no way of confirming this. A phase error introduced by a misplaced feed would have reduced gain and raised the sidelobe level in the forward hemisphere, but would not have introduced sidelobes in any particular direction. We note that the absolute calibration process can be relied upon to give the correct answer, whatever aperture efficiency was achieved.

The error in our measurement of aperture efficiency, and consequently in the intensity scale of our data, is probably on the order of $5 \%$. To this must be added a further $5 \%$ for the probable error in the adopted flux density of Taurus A, yielding an overall accuracy for the amplitude scale of $7 \%$.

Knowing the aperture efficiency allowed us to convert the survey data to antenna temperature, $T_{A}$, but the desired product from the survey is brightness temperature, $T_{B}$. The two are related by

$$
T_{B}=\frac{\Omega}{\Omega_{B}} T_{A} .
$$

Given that the telescope beams are closely Gaussian, $\Omega_{B}$ was taken to be the solid angle of a Gaussian of half-power width, $\theta$, equal to the measured beamwidth (see Figure 7). Then $\Omega_{B}=1.13 \theta^{2}$.

\section{Data Processing}

Raw data were recorded in the RPFITS format, with each file containing a single azimuth scan or raster map. These files were converted into SDFITS, using the program rp2sdfits of the ATNF livedata library, and finally converted into our own binary format for further calibration and processing with $\mathrm{C}++$ code written especially for this survey. We will now discuss the particulars of most of the data processing steps in the order applied.

\subsection{Primary Calibrators}

At least once a night, one of two primary calibrators (Fornax-A and Pictor-A) was observed, usually around sunset or sunrise, depending on the time of year. Observations were made as raster scans resulting in a rectangular map centered on the source. Scan separation was $12^{\prime}$, yielding greatly oversampled maps at the low end of the band. A two-dimensional Gaussian function was fitted to each of these maps, with the free parameters allowing for an elliptical and tilted beam. The derived amplitude was corrected for atmospheric attenuation (a small effect, $<0.1 \%$ ). Both of these sources were slightly resolved by our beams, but that is not a concern here, because we were using only the amplitude of the fitted Gaussian.

Using this database of Gaussian fits, an average flux density and temperature spectral index was determined for the two calibrators (Fornax-A: -2.5 , Pictor-A: -2.1 ). This is by no means an absolute flux calibration; it was used only to remove gain and phase drifts across time, to remove the instrumental frequency response from the data, and to correct on-axis instrumental polarization.

\subsection{Noise Source Calibrations}

The noise source provided a polarized and stable signal. It was switched on every $2-3 \mathrm{hr}$ for a duration of 5 minutes, alternating between $\mathrm{ON}$ and $\mathrm{OFF}$ with a frequency of $1 \mathrm{~Hz}$. The resulting $\mathrm{OFF}$ measurements were averaged and subtracted from the average of the $\mathrm{ON}$ measurements, providing noisesource temperatures for all four correlation outputs $\left(R R^{*}, L L^{*}\right.$, $R L^{*}$, and $\left.L R^{*}\right)$ at each frequency channel. The time series of these measurements was smoothed by averaging and discarding outliers, allowing us to detect and correct gain variations of the receiver between calibrations. All data were divided by the smoothed series with interpolation between measurements, resulting in data now calibrated in units of noise-source temperature.

\subsection{Instrumental Polarization}

Two effects contribute to instrumental polarization: (a) cross-hand leakage between $L$ and $R$ channels occurring in the feed, the phasing network, and (perhaps) in the receiver; and (b) cross-polarization in the feed radiation characteristics. We have corrected on-axis polarization, but have not dealt with the much more complex problem of cross-polarization in the antenna response.

In the absence of instrumental polarization, a scan across an unpolarized source would produce a Gaussian-like profile in total intensity channels $L L^{*}$ and $R R^{*}$ and no signal in the two cross-hand channels $L R^{*}$ and $R L^{*}$. In fact, $L R^{*}$ and $R L^{*}$ showed complicated structure, a combination of effects (a) and (b). Using an iterative process, we found factors $f_{U}$ and $f_{Q}$ for each channel, which minimized the response in $L R^{*}$ and $R L^{*}$. The "unpolarized" source used was Fornax A. (Although the source is a synchrotron emitter whose emission must be polarized, when the entire source was averaged in our large beams, it became essentially unpolarized). The ratio at the beam center of $L R^{*}$ and $R L^{*}$ to total intensity was determined for every Fornax A observation. These values were taken as the best estimate of the on-axis instrumental polarization. Smoothing in time was applied to these values, because rapid variations of instrumental polarization were not expected. The factors $f_{U}$ and $f_{Q}$ were stable with time within $\pm 10 \%$. Instrumental polarization artifacts remain in the data along the Galactic plane, where $I$ emission is very strong (see Figures 19 and 20). Small polarization artifacts, the classical "four-leaf clover" response, remain in the $Q$ and $U$ data around strong compact sources at a level of a few percent, and there is some variation from one such source to another. The portrayal of the low-level extended emission, the objective of this survey, is not impaired by these very localized blemishes.

\subsection{Ground Radiation}

The observing technique, azimuth scanning, assumes that emission from the ground is constant with azimuth. In the frequency range of this survey, that assumption may not be quite true. The far sidelobes of the telescope, including the spillover sidelobes, have very strong instrumental polarization (e.g., Du et al. 2016). They convert unpolarized signal from the ground (or from the sky) into apparently polarized signal. In the range $300-480 \mathrm{MHz}$, the ground is definitely not a blackbody; it is a partly polarized emitter (Du et al. 2016), and it will also reflect sky signal into the spillover sidelobes. We estimated the ground contribution to the data by plotting all scans against 


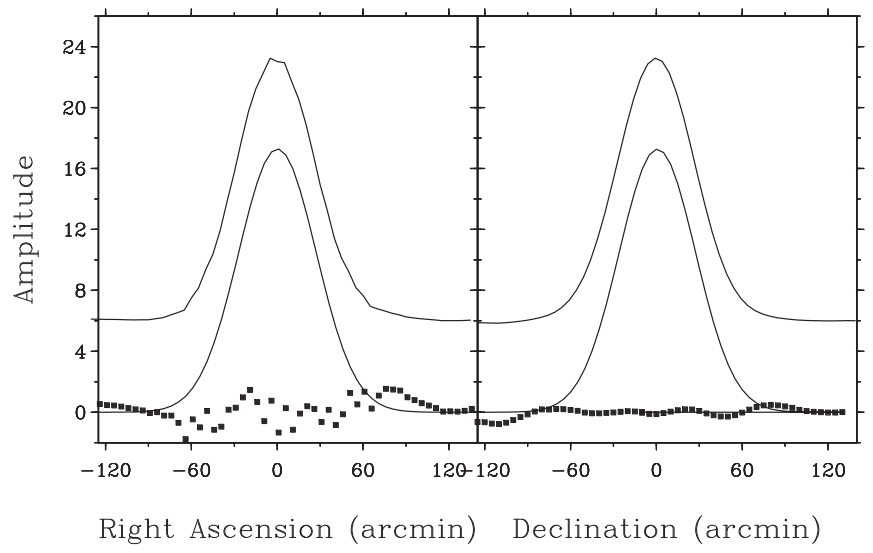

Figure 6. Cross sections in R.A. and decl. through the telescope beam at 365.75 MHz. The amplitude units are arbitrary. The top curves, displaced vertically by six units, show the measured data. The lower curves are Gaussians, fitted to the data. The dotted curves show residuals, multiplied by five for clarity. Scanning was in the decl. direction, and some scan-to-scan variations appear as additional residuals in the R.A. scan.

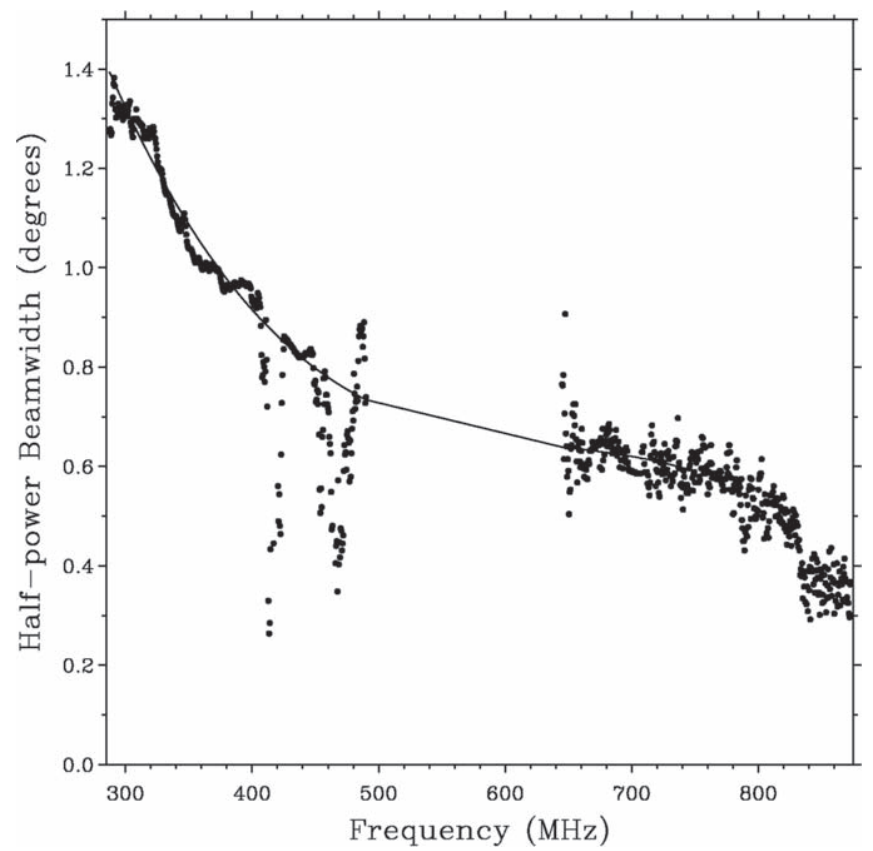

Figure 7. The beamwidth of the telescope, measured from raster scans of Taurus A. Twelve separate scans made on different days throughout the survey period were averaged to produce the data in this figure. The presence of RFI is obvious. Fluctuations are higher above $660 \mathrm{MHz}$, probably due to RFI, and no data from that band were used in this calibration. A cubic polynomial, fitted to the data up to $750 \mathrm{MHz}$, is shown; this curve was used in absolute calibration of the survey. The rapid fall in deduced beamwidth above $\sim 800 \mathrm{MHz}$ is not completely understood (see text).

azimuth and masking out emission from the Galactic plane. We binned these data in steps of $20^{\circ}$ in azimuth, found the median in each bin, and interpolated between these points with a spline function. This was done independently for each frequency channel, and the fitted functions were subtracted from the data. The corrections were $0.2 \mathrm{~K}$ or less.

\subsection{Radio Frequency Interference}

RFI posed a major threat to this survey. Locally generated RFI was minimized by turning off fluorescent lights, computers

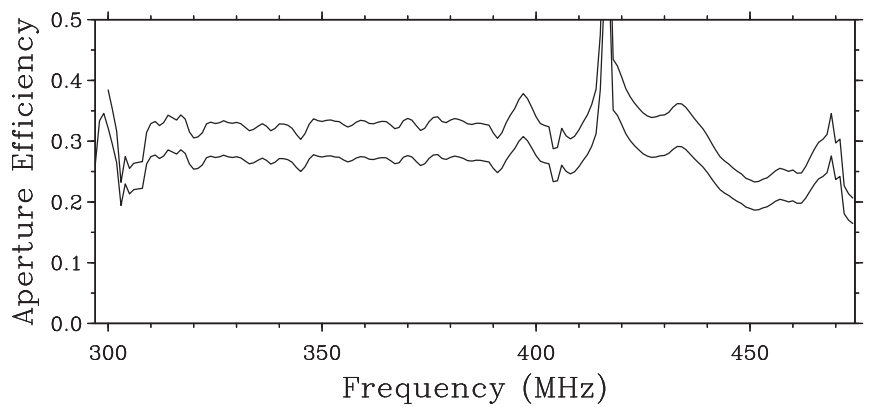

Figure 8. The lower curve shows the measured aperture efficiency of the telescope. The best estimate of aperture efficiency is a constant value of $\eta_{A}=0.27 \pm 0.01$ across the band (see text). The values shown here include losses in the feed and phasing network. The upper curve shows the aperture efficiency corrected for those losses (see Figure 5).

and other equipment (unused during our night-time observations) in all buildings around the telescope. A monitoring system in another receiver in the focus cabin was also shut down every night.

The band $500-650 \mathrm{MHz}$ was totally occupied by digital television signals broadcast from mountaintops 100 and $200 \mathrm{~km}$ distant from the Observatory. The band $490-660 \mathrm{MHz}$ was therefore completely removed from the receiver passband with filters, and no attempt was made to observe within it. The receiver passband for the survey was determined by bandpass filters as $285-485 \mathrm{MHz}$ and $660-870 \mathrm{MHz}$, which we refer to as the lower and upper bands respectively. RFI, both steady and intermittent, occurred in both lower and upper bands: in the upper band, we lost $80 \%$ of our observations to RFI; in the lower band, we lost 50\%. Data loss in the upper band was so severe that we have not yet been able to use the data in that frequency range. We have been able to use data in the lower band because the sky was heavily oversampled relative to the telescope beam in that band. The sampling interval was set at 10.5 , just under half the beamwidth at $900 \mathrm{MHz}$. This sampling interval is about one fifth of the beamwidth at $300 \mathrm{MHz}$ and one third of the beamwidth at $480 \mathrm{MHz}$ : at the low end of the band, the sky was oversampled, and sky sampling is still adequate even after removing RFI-affected data. We present data to a lower frequency limit of $\sim 287 \mathrm{MHz}$, but frequency channels below $300 \mathrm{MHz}$ are heavily affected by RFI.

Our RFI mitigation strategy depended heavily on oversampling in time and frequency. The first step of RFI mitigation consisted of flagging outliers in a time series. Every pixel was covered by many observations, spaced over $3.5 \mathrm{yr}$, and RFI is time-variable, especially over such a long period. For every pixel, we assembled a time series of observations and calculated the median and standard deviation for that series. Every integration above or below the median by about three standard deviations was flagged (flagging level varied a little with frequency, and flagging level was set higher in regions of high sky brightness). Median and standard deviation were calculated again, and more outliers flagged; the process was repeated until no integrations lay outside the thresholds. The heavy spatial oversampling allowed us to set low thresholds for RFI flagging.

The second step of RFI mitigation examined the data after processing to find outliers along the frequency axis. A spectral index was determined for every pixel, and a baseline subtracted from the spectrum. All channels above or below a certain threshold were flagged. Figure 9 shows the fraction of pixels 


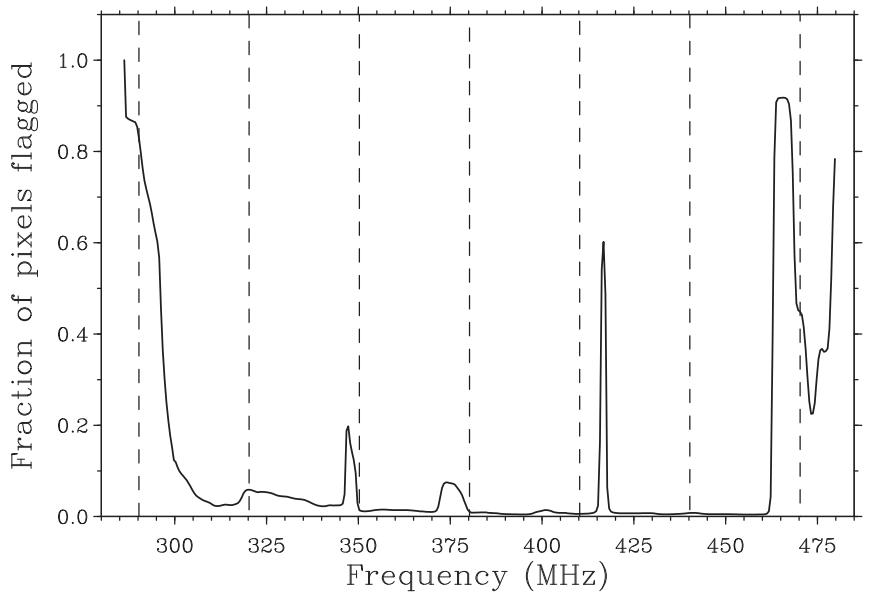

Figure 9. The fraction of the survey data flagged because of RFI, shown as a function of frequency. The vertical dashed lines indicate the frequencies used in the calculation of the T-T plots shown in Figure 14.

flagged in the final data product. This number is low in most frequency channels, attesting to the effectiveness of oversampling. That being said, the final data products are not noiselimited: they are probably limited by RFI at even lower levels.

\subsection{Ionospheric Faraday Rotation}

High electron densities in the ionosphere and a geomagnetic field component along the line of sight give rise to Faraday rotation whose magnitude is highly variable and dependent on solar activity. Observations for this survey were made between sunset and sunrise, but spanned a period from solar minimum in 2009 to one where the Sun was fairly active in mid-2012. The ionospheric RM ranged from zero to about $-4 \mathrm{rad} \mathrm{m}^{-2}$. Because $1 \mathrm{rad} \mathrm{m}^{-2}$ rotates the polarization angle by one radian at $300 \mathrm{MHz}$, this obviously had to be corrected.

Ionospheric Faraday rotation was corrected using an algorithm based on the International Reference Ionosphere model (Bilitza 2015) and a static model of the geomagnetic field (Thébault et al. 2015). The input quantity is the $10.7 \mathrm{~cm}$ solar flux (Tapping 2013). The model computes Faraday rotation through the ionosphere as a function of direction. Figure 10 shows the predicted ionospheric Faraday rotation through the course of the survey.

More sophisticated routines are now available for calculation of ionospheric RM (e.g., Mevius 2018), using GPS data to calculate the total electron content of the ionosphere. From the $\sim 50,000$ corrections that we computed (Figure 10), we selected 53 representative samples. We made new calculations for those dates and telescope pointings using RMextract and the ALBUS code (A. G. Willis et al 2019, in preparation), respectively. The average ratio between RMextract and our calculations was 1.155; the average ratio with ALBUS was 1.005. We regard ALBUS as the superior code in the Australian context because it uses many more GPS stations than RMextract. For $|\mathrm{RM}|<1.0$, the peak difference between ALBUS and our calculations was $0.2 \mathrm{rad} \mathrm{m}^{-2}$, and the rms difference was 0.07 . For $1.0<|\mathrm{RM}|<3.0$, the peak difference was $0.6 \mathrm{rad} \mathrm{m}^{-2}$ and the rms difference was 0.2 . We found one value where the GMIMS RM was $4 \mathrm{rad} \mathrm{m}^{-2}$ and ALBUS gave $5 \mathrm{rad} \mathrm{m}^{-2}$ : this is the very highest RM in the set of 50,000 calculations, and we consider it an outlier.
In this survey, measurements taken at different times were combined (vector averaged) using the basketweaving technique (see Section 5.7). Errors in the ionospheric RM correction could have led to a reduction in the measured polarized intensity below the true value-affecting, for example, estimates of the fractional polarization (see Figure 18). An error of $0.2 \mathrm{rad} \mathrm{m}^{-2}$ in the correction applied to two polarization vectors that are then averaged leads to a reduction in estimated polarized intensity of $2 \%$ at $300 \mathrm{MHz}$ and correspondingly less at higher frequencies. This is a small effect.

\subsection{Basketweaving}

The observing technique, making many intersecting scans, has been described in Section 3. Basketweaving, the reconciliation of intersecting scans, was an integral part of the processing; our implementation mostly followed the procedure of Haslam et al. (1981).

The correlator output was the combination of several sources, receiver noise, ground and spillover noise, and sky signal. While the sky signal changes with position, the other contributions should remain constant. In reality, however, any one of the system noise contributions may vary over time, caused by gain variations of the receiver or by actual, intrinsic variations of the signal (e.g., variations of ground reflectivity with soil moisture). The challenge in data processing was to separate the sky signal from the rest, and to subtract that baseline with minimal effect on the sky signal. The first step in processing was to remove a linear baseline from each scan. The effect was specific to the various correlator outputs. From the total intensity products $L L^{*}$ and $R R^{*}$, this removed ground radiation, assumed constant during the scan, and it removed system noise, likely to be highly stable over the duration of one scan, but possibly variable on longer timescales; it also removed some signal. From the polarization products $L R^{*}$ and $R L^{*}$, this subtraction removed the ground radiation and the instrumental polarization component, and the small component of receiver noise that coupled between $L$ and $R$ via polarization leakage, but removed very little, if any, sky signal. The long scans in azimuth with the feed at a fixed orientation modulated the sky polarization by the parallactic angle (Carretti et al. 2019 ), and the average of sky signal in each $L R^{*}$ and $R L^{*}$ scan was always close to zero: $L R^{*}(=Q)$ and $R L^{*}(=U)$ tend to average to zero along long scans, especially at the low frequencies of our survey. Parallactic angle rotation was then applied, restoring very closely correct zero levels to $L R^{*}$ and $R L^{*}$.

Basketweaving was performed on every channel individually, and separately on all four products, $L L^{*}, R R^{*}, L R^{*}$, and $R L^{*}$. Baseline fitting ran through several loops. The first two loops fitted constants to each baselevel. For the next few loops, the differences were smoothed over ten degrees, and then over five degrees for two final loops. We also detected low-level periodic variations. We suspect these arose from gain variations of the amplifiers that were not corrected by the noise source calibration. These baseline fluctuations were removed by fitting sinusoids.

In the final analysis, our data are not limited by thermal noise, but probably by low-level RFI (Section 5.5). The baseline fitting procedure that we adopted removed some RFI. It could possibly have been taken further, but at the risk of removing some real signal. Basketweaving did remove the sky 
Table 2

Available Data Products

\begin{tabular}{|c|c|c|c|c|c|}
\hline Data Product & $\begin{array}{l}\text { Beam } \\
(\text { deg) }\end{array}$ & $\begin{array}{l}\text { Coverage } \\
(\mathrm{MHz})\end{array}$ & $\begin{array}{l}\text { Freq. Resolution } \\
(\mathrm{MHz})\end{array}$ & $\begin{array}{l}\text { FD Increment } \\
\left(\operatorname{rad~m}^{-2}\right)\end{array}$ & $\begin{array}{l}\text { FD Coverage } \\
\left(\mathrm{rad} \mathrm{m}^{-2}\right)\end{array}$ \\
\hline Total intensity & 1.35 & $286.25-487.75$ & 0.5 & $\ldots$ & $\ldots$ \\
\hline Stokes $Q$ and $U$ & 1.35 & $286.25-487.75$ & 0.5 & $\cdots$ & $\cdots$ \\
\hline Polarized intensity & 1.35 & $286.25-487.75$ & 0.5 & $\cdots$ & $\cdots$ \\
\hline Polarization angle & 1.35 & $286.25-487.75$ & 0.5 & $\cdots$ & $\cdots$ \\
\hline Faraday depth cube & $\cdots$ & $\cdots$ & $\cdots$ & 0.5 & -100 to +100 \\
\hline
\end{tabular}
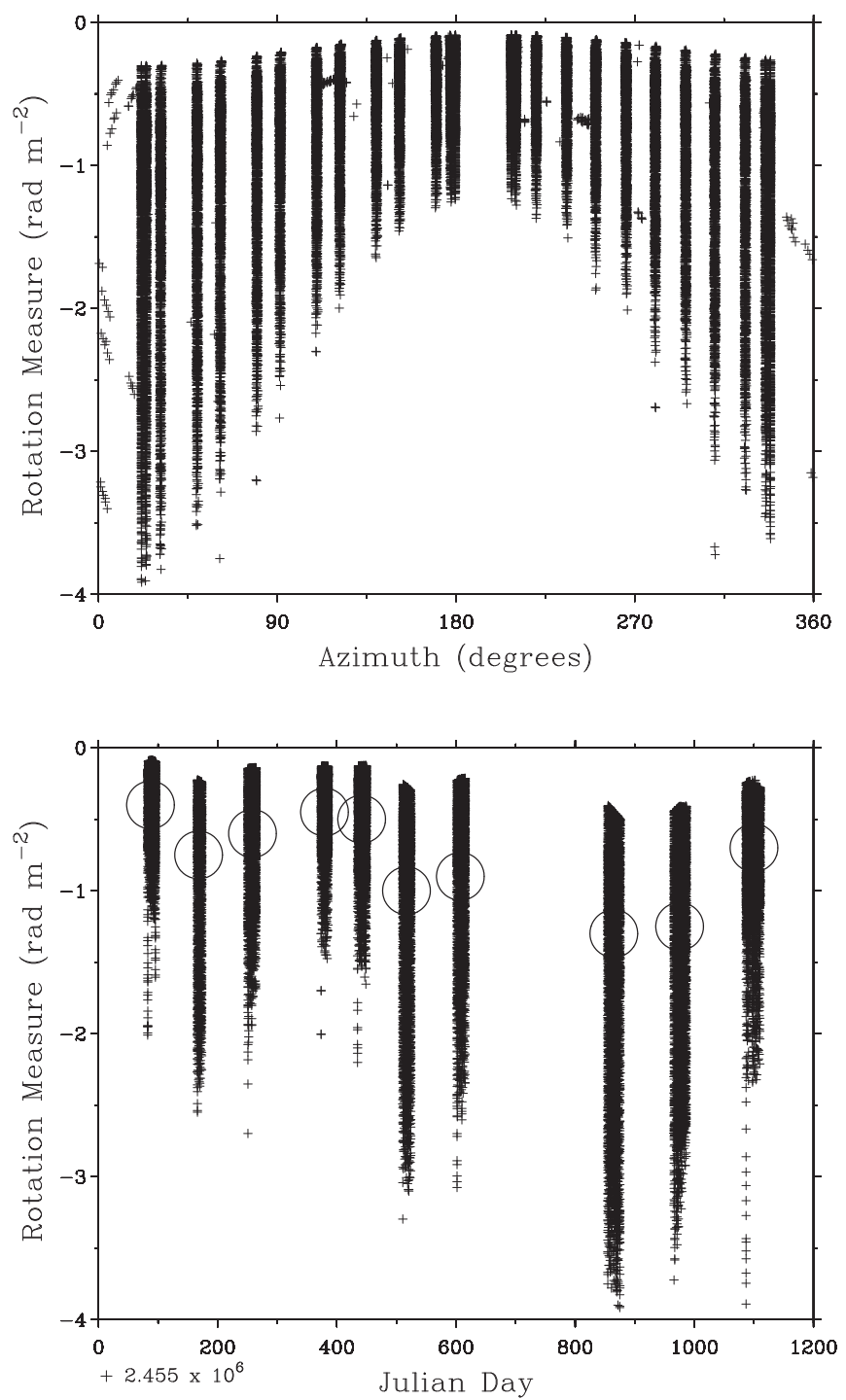

Figure 10. Top: predicted ionospheric Faraday rotation for all observations as a function of azimuth. Faraday rotation was calculated at the azimuths shown, and interpolated values were used for intermediate pointings. Bottom: ionospheric Faraday rotation as a function of Julian day. The ten blocks of data correspond to ten observing sessions (see Table 1). Circles indicate median values in individual observing sessions.

minimum from the $L L^{*}$ and $R R^{*}$ images, and any use of the Stokes $I$ data product will have to take that into account.

\subsection{Gridding and Smoothing}

To this point, the data have remained in the form of scans, long azimuth tracks across the equatorial grid. Maps in equatorial and Galactic coordinate frames were made from the scans by a simple gridding process: scan values falling within a square with sides one grid interval, centered on each equatorial grid point, were averaged. Smoothing to the final angular resolution, $1^{\circ} .35$, was done at this point.

\subsection{RM Synthesis}

As described by Brentjens \& de Bruyn (2005), a survey of linear polarization that covers a sufficiently broad range of frequency, or, more exactly, of wavelength squared $\left(\lambda^{2}\right)$, can be inverted by a Fourier transform to Faraday depth space $(\phi)$. Because of the many interfering signals that required flagging of some or all data at specific frequencies, it is necessary for the Fourier transform routine employed in this operation to handle missing data intelligently. Our starting point was a cube of $360 Q$ and $U$ images evenly spaced in frequency, spanning 300.25 to $479.75 \mathrm{MHz}$ in steps of $0.5 \mathrm{MHz}$, with each channel smoothed to the angular resolution (1.35) at the lowest frequency. From these data, we computed Faraday depth cubes in $Q$ and $U$ covering $-100<\phi<+100 \mathrm{rad} \mathrm{m}^{-2}$ in steps of $0.5 \mathrm{rad} \mathrm{m}^{-2}$. Our RM synthesis routine ${ }^{24}$ implemented the equations of Brentjens \& de Bruyn (2005), programmed in python. All frequencies were weighted equally.

The data cube emerging from this process represents sky emission convolved with a Rotation Measure Spread Function (RMSF) that is approximately the Fourier transform of the sampling in $\lambda^{2}$. In a perfect world, this sampling would be complete and uniform, and deconvolution would be simple. In reality, however, our heavy flagging of RFI-affected data produced RMSFs that had strong sidelobes and differed from one pixel to the next. RMSFs were therefore calculated separately for each pixel. The "dirty" $\phi$ spectra were deconvolved with the RMCLEAN routine (Heald 2009). Loop gain was 0.1 and the process was limited to $60 \mathrm{mK}$ (the survey rms) or 1000 iterations. The Faraday depth spectrum at each pixel was restored using a Gaussian function fitted to the RMSF for that pixel. Finally, residuals were added back. The parameters of the resulting data are listed in Tables 1 and 2 .

Figure 11 shows RMSFs relevant to this data set. The primary effect of the flagged channels on the RMSF is to generate sidelobes at a level of $\sim 4 \%$ at $\pm 90 \mathrm{rad} \mathrm{m}^{-2}$. The median RMSF (median in width of the main lobe) is close to the narrowest RMSF, indicating that flagging of RFI in the spectra retained in the data set was quite modest over much of the sky. Figure 12 shows both dirty and clean Faraday spectra for three positions, each illustrating a different situation. Panel (1) shows a simple spectrum from an apparently Faraday-thin region. The dirty spectrum has roughly symmetrical sidelobes

\footnotetext{
${ }^{24}$ RM_tools_3D, version of 2018 January 31, available from https://github. com/crpurcell/RM-tools.
} 


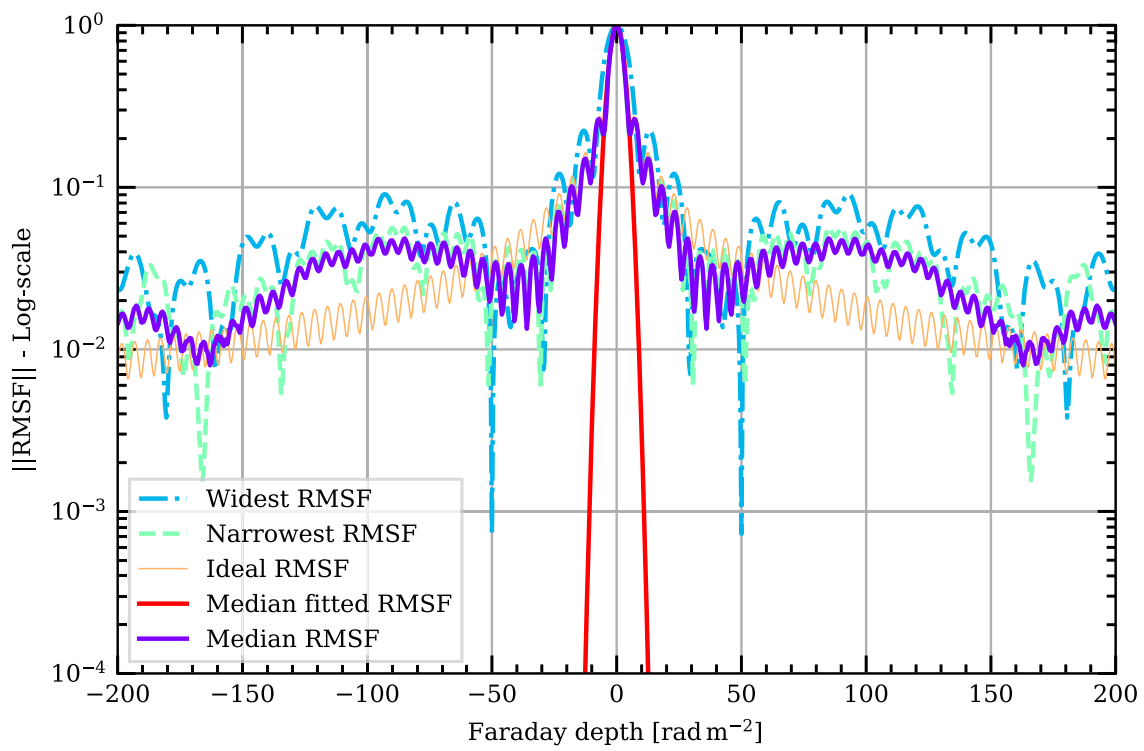

Figure 11. The Rotation Measure Spread Functions (RMSFs) of this data set, shown over twice the range in Faraday depth of the final data set. The narrowest, widest, and median RMSFs are shown, together with the ideal RMSF, as it would be if no frequency channels were flagged.
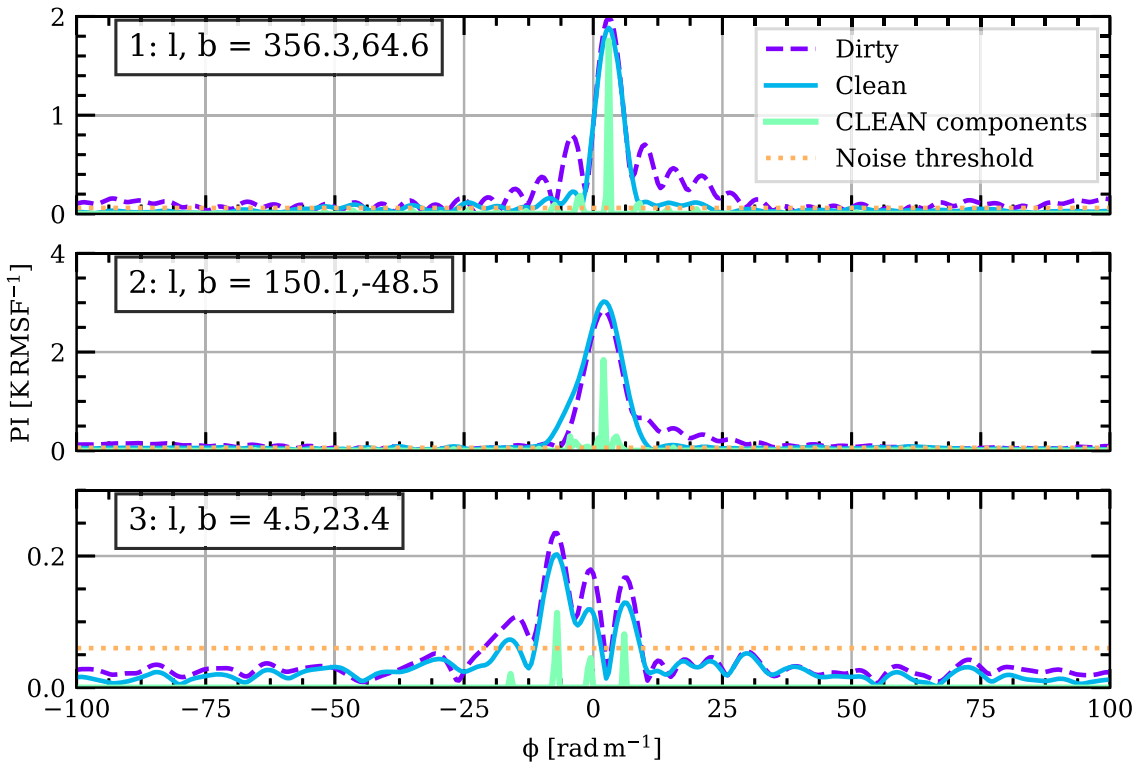

Figure 12. Faraday depth spectra for three lines of sight. The position in Galactic coordinates is indicated in each panel. See text for discussion of these spectra.

on either side of the main peak; it is satisfactorily fitted by a single clean component, and the sidelobes are almost completely removed by the cleaning process. Panel (2) shows a single feature, broader than the RMSF, with a long tail toward positive $\phi$. It is striking that the clean spectrum shows a tail toward negative $\phi$ (and the sidelobes are almost completely removed). Panel (3) shows a low-intensity complex spectrum. Note that the relative heights of the three peaks in the spectrum are changed by cleaning. However, the significance of this change should not be overemphasized: such occurrences are a common manifestation of the complex RMCLEAN algorithm (Sun et al. 2015b).

\section{Quality of the Survey Data}

In order to assess the quality of the survey data, we made comparisons with existing data to the extent possible. Although the data presented here were calibrated independently, comparison with other data helps with estimation of errors.

\subsection{The Intensity Scale}

The first step was to test the calibration of the total-intensity (Stokes parameter $I$ ) scale. We used the T-T plot technique (Costain 1960), in which the brightness temperatures, $T_{B}$, at sky directions in one data set are plotted against the brightness temperatures in the same directions from another data set. If two data sets at the same frequency are compared in this way, the slope of the straight line fitted to the points gives the ratio of the two intensity scales. If the two data sets are at different frequencies, the slope of such a plot gives the temperature spectral index, $\beta$, defined by $T_{B} \propto \nu^{\beta}$, in that frequency interval (affected by any errors in the intensity scales). 


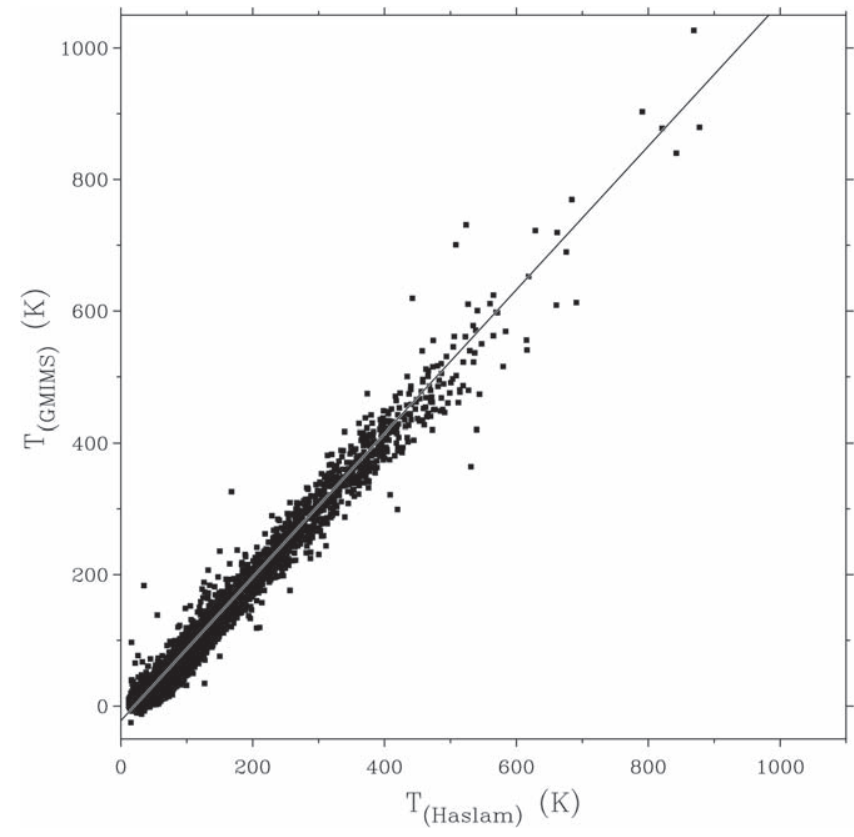

Figure 13. Brightness temperature at $408 \mathrm{MHz}$ plotted point-by-point against brightness temperature from the Haslam et al. (1982) survey at the same frequency. The plot includes data from the entire GMIMS survey area. Bandwidth in both cases is $3.5 \mathrm{MHz}$ and beamwidths are $51^{\prime}$. The fitted line has a slope of 1.09 and an offset of $-19 \mathrm{~K}$.

We plotted total GMIMS intensity at $408 \mathrm{MHz}$ against corresponding values from the survey of Haslam et al. (1982). We used data at the resolution of the telescope, $51^{\prime}$ in both cases. Figure 13 shows the result. There are two conclusions. First, the fitted line shown in Figure 13 indicates that the GMIMS intensity scale is $9 \%$ higher than the Haslam et al. (1982) scale. That is a satisfying result, given that the two surveys were independently calibrated to absolute scales. Second, the T-T plot shows that the GMIMS zero level is about $19 \mathrm{~K}$ lower than the zero of the Haslam data. This is expected, because the basketweaving process has removed the data minimum; the sky minimum in the Haslam data is about $13 \mathrm{~K}$ (and the quoted error in the zero level is $\pm 3 \mathrm{~K}$ ).

We cannot say much about this $9 \%$ difference, except that it is satisfyingly small. A direct comparison of our methods with the methods employed by Haslam et al. (1982) is difficult. The Haslam et al. (1982) survey was calibrated by adjusting levels to agree with the earlier survey of Pauliny-Toth \& Shakeshaft (1962). The 1962 survey covered the sky north of decl. $-20^{\circ}$, and the calibration factors established by Haslam et al. (1982) for the northern sky had to be extrapolated to the south, creating an uncertainty (over most of the area of our survey) that we cannot evaluate. Furthermore, the Haslam et al. (1982) data were tied to the main-beam brightness temperatures from Pauliny-Toth \& Shakeshaft (1962), eliminating steps of establishing main beam solid angle or beam efficiency for the 1982 data. Again, we cannot compare methods. Nevertheless, the Haslam et al. (1982) survey is well-regarded, and its calibration is considered strong. It is a satisfying outcome that the two intensity scales agree so well.

Having satisfied ourselves that the intensity scale at $408 \mathrm{MHz}$ is correct, we needed to check the scale at other frequencies across the band. We chose seven frequencies from 290.25 to $470.25 \mathrm{MHz}$ at intervals of $30 \mathrm{MHz}$ (marked in
Figure 9). We averaged five channels at each of these frequencies, to give bands of width $2.5 \mathrm{MHz}$. From these, we generated six T-T plots (shown in Figure 14) between pairs of frequencies using all the data points in the survey, i.e., over the entire southern sky. The slope of these T-T plots reflects the spectrum of the extended Galactic emission. In this frequency range, the emission is predominantly nonthermal, with relatively little contribution from optically thin thermal gas.

If we know, or assume, a spectral index for the emission, we can check the intensity scales at frequencies other than $408 \mathrm{MHz}$. Data from the northern sky indicate $\beta \approx-2.5$ between 151 and $408 \mathrm{MHz}$ (Sironi 1974) and $\beta \approx-2.8$ between 408 and $1407 \mathrm{MHz}$ (Webster 1974). Data for the southern sky between 408 and $720 \mathrm{MHz}$ give $\beta \approx-2.8$ (Landecker 1969).

The correlations in Figure 14 are tight: correlation coefficients are above 0.994 for $\mathrm{T}-\mathrm{T}$ plots from 320.25 to $440.25 \mathrm{MHz}$. Correlation coefficients of T-T plots involving the outer frequencies, 290.25 and $470.25 \mathrm{MHz}$, are not quite as high because there was considerable RFI in these bands and many sky points were intentionally flagged (at $290.25 \mathrm{MHz}$ $86 \%$ of data was flagged, and at $470.25 \mathrm{MHz} 60 \%$ was flagged). The temperature spectral indices deduced from the $\mathrm{T}-\mathrm{T}$ plots in Figure 14 vary from $\sim-0.9$ to $\sim-3.7$, but the frequency span for each calculation is very small, and small inaccuracies in the temperature scales will produce large discrepancies in deduced spectral indices. We calculated correction factors for each of the six $\mathrm{T}-\mathrm{T}$ plots required to bring $\beta$ to a value of -2.8 : these range from 0.994 to 1.178 .

However, this is not a very strong discriminator. If we choose any value of $\beta$ between -2.4 and -3.2 for this frequency range, the correction factors calculated as above are still in the range 0.9-1.1. All considered, we conclude that the intensity scale across $300-480 \mathrm{MHz}$ is correct within $10 \%$. This calibration of total intensity immediately implies that polarized intensities are correct to the same accuracy.

\subsection{Calibration of Polarization Angle}

In an attempt to calibrate polarization angle, we interrupted observing at two-hour intervals to make a rotating-feed observation of one of a number of highly polarized regions. These regions were chosen on the basis of the data of Mathewson \& Milne (1965), a $408 \mathrm{MHz}$ polarization survey made with the Parkes Telescope. These calibrations were unsuccessful. We believe that distant sidelobes passing over bright unpolarized regions when the feed was rotated generated variations stronger than those arising from the polarized region at beam center. Perhaps the feed used for this survey was not as highly tapered as the feed used by Mathewson \& Milne (1965). See Section 5.4 for a discussion of the properties of distant sidelobes.

In the face of this failure, we fell back on a post-observation comparison of polarization angles with the data of Mathewson \& Milne (1965). We compared the two data sets over most of the southern sky, giving the most weight to regions of high polarized emission. From this comparison, we deduced that a correction of $-60^{\circ}$ should be applied to the GMIMS data, and this correction has been applied to the released data. In Figure 15, we show the comparison after the correction. We then made similar comparisons with the 408 and $465 \mathrm{MHz}$ data from Brouw \& Spoelstra (1976); these surveys extend only to 


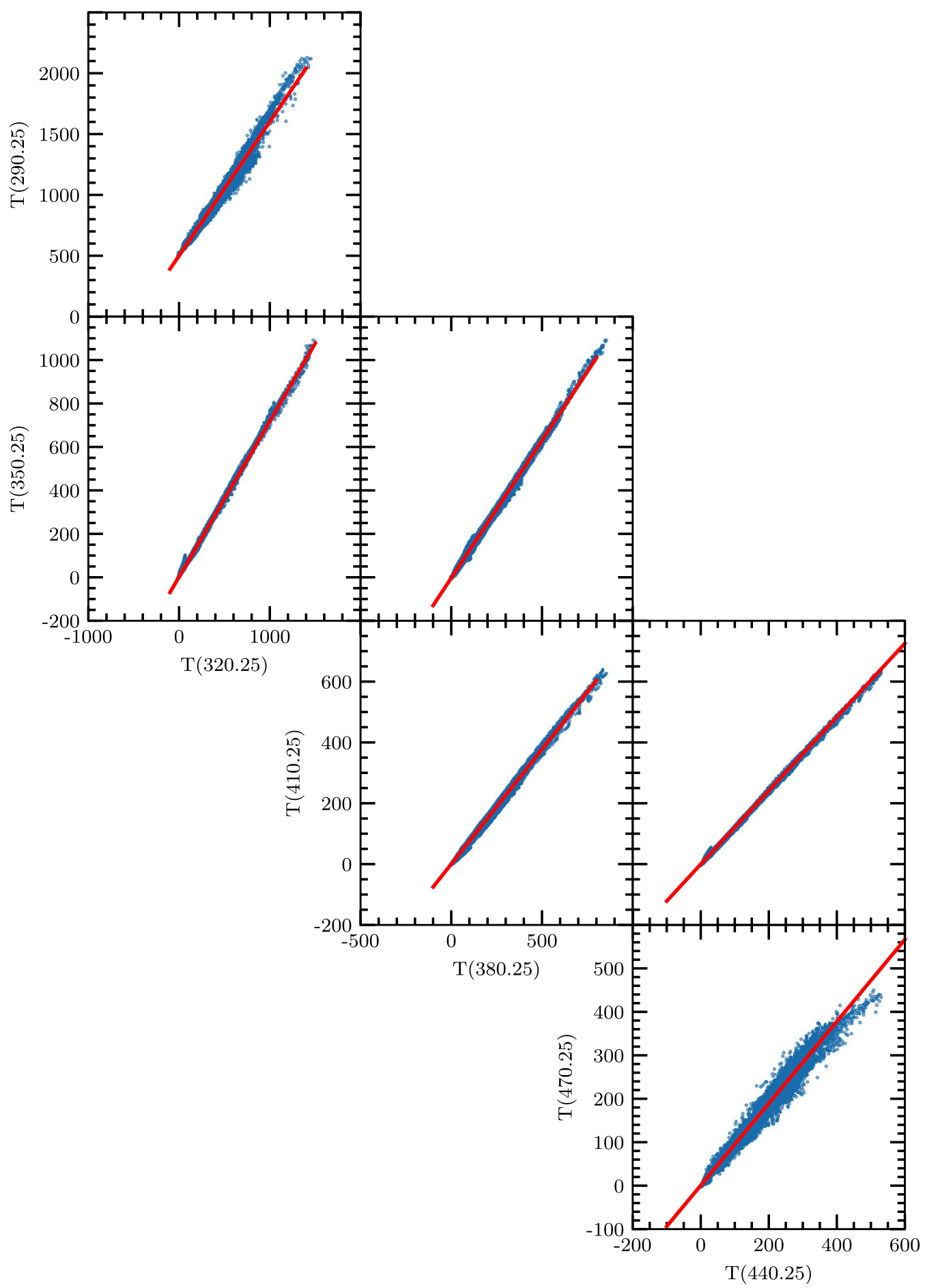

Figure 14. Spectral index of total intensity emission measured by plotting brightness temperature at one frequency against temperature at the corresponding point measured at the second frequency. Plots are shown for six pairs of frequencies. See text for interpretation of these results.

decl. $0^{\circ}$, so the comparisons included fewer points. The results are shown in Figures 16 and 17.

The data of Mathewson \& Milne (1965) were obtained with a rotating dipole feed, for which the zero is mechanically defined and should be absolute. However, this does not ensure correct calibration of their final data because a substantial correction for ionospheric Faraday rotation was applied and the angles were then adjusted with measurements of reference regions taken from earlier data from the Dwingeloo Telescope, data which subsequently went into the data published by
Brouw \& Spoelstra (1976). In other words, the comparisons in Figures 15 and 16 are not entirely independent. The fits illustrated by straight lines plotted in Figures 15-17 may indicate corrections slightly different from $-60^{\circ}$. However, these fits give equal weight to all data points; when the comparison is made with the points where polarized intensity is highest, the offset of $-60^{\circ}$ emerges very clearly. Although the comparisons were limited to 408 and $465 \mathrm{MHz}$, there does not seem to be a frequency-dependent correction. While there is no further check possible using independent data, our data alone 


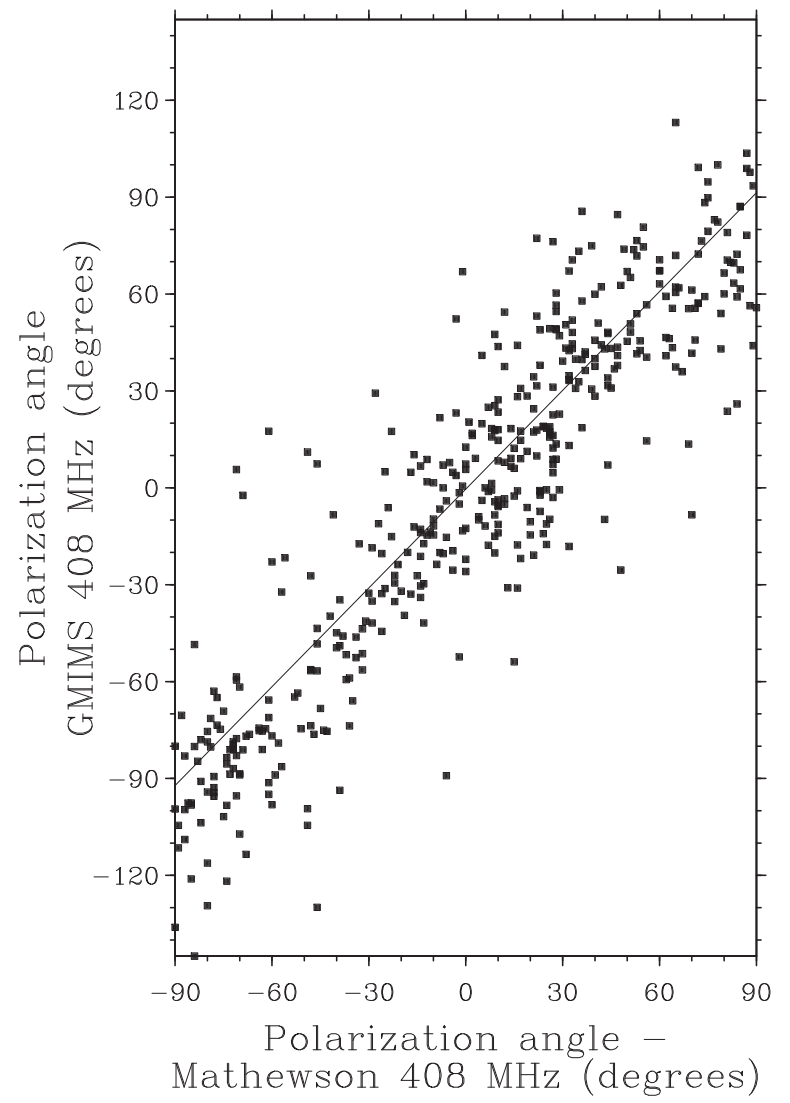

Figure 15. Comparison of polarization angle at $408 \mathrm{MHz}$ between the present work and the survey of Mathewson \& Milne (1965). The values for 437 points over the entire southern sky common to the two surveys are shown. We have subtracted $60^{\circ}$ from the raw GMIMS values, and for clarity, the GMIMS values have been permitted to fall outside the range $\pm 90^{\circ}$. The solid line shows a fit to all points. The slope of the line is 1.02 .

do provide us with assurance that the angles are close to correct. Inspection of the Faraday depth cube after RM Synthesis shows that, over most of the sky, the strongest emission is at a Faraday depth of $0 \pm 0.5 \mathrm{rad} \mathrm{m}^{-2}$ and the allsky average is very close to $0 \mathrm{rad} \mathrm{m}^{-2}$, as would be expected.

\section{Results}

Detailed analysis of the survey data is beyond the scope of this paper, and this section presents only very general conclusions. The survey parameters are summarized in Table 1. The data from this survey are available from the Canadian Astronomy Data Centre. ${ }^{25}$ Available data products are listed in Table 2.

The data products include total-intensity maps. They portray the sky as seen in earlier surveys (in particular, the survey of Haslam et al. 1982). We do not show any total-intensity data here, as such a figure is unlikely to convey much new information. The basketweaving process (see Section 5.7) inevitably removes the sky minimum from the total-intensity data, so the zero level of these images is not correct, but the intensity scale is absolutely calibrated at all frequencies. What is new is the extensive frequency coverage, and we can expect that the data do carry new information on spectral index, which might be extracted with the T-T plot technique (see Figure 14).

25 doi: $10.11570 / 18.0007$

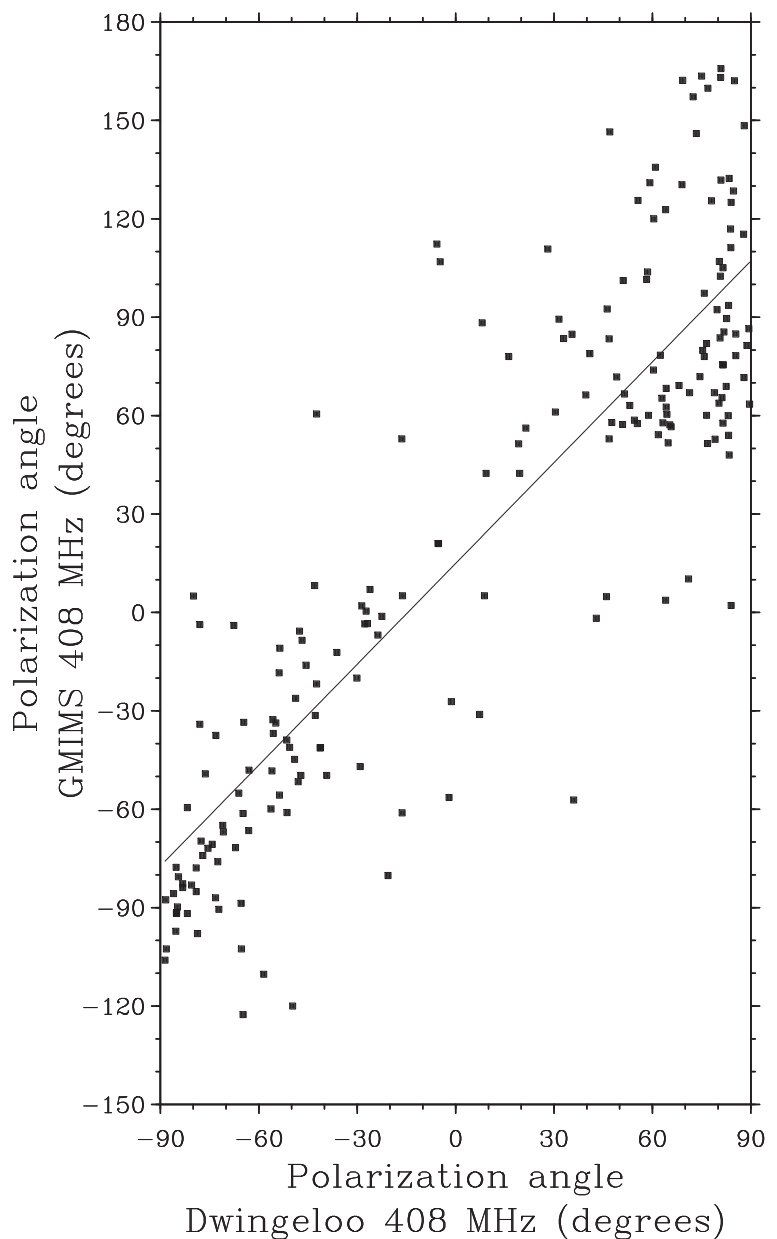

Figure 16. Comparison of polarization angle between the present work and the $408 \mathrm{MHz}$ survey of Brouw \& Spoelstra (1976). The comparison is confined to positions where polarized intensity exceeds $1 \mathrm{~K}$. The area of overlap is between decl. $0^{\circ}$ and $+20^{\circ}$, containing 193 common points. $60^{\circ}$ has been subtracted from the raw GMIMS values, and the GMIMS values have been allowed to fall outside the range $\pm 90^{\circ}$. The solid line shows a fit to all points. The slope of the line is 1.02 .

A detailed study is beyond the scope of this paper. However, it is already evident from the plots in Figure 14 that the emission in this frequency range is predominantly synchrotron emission. If significant thermal emission were present, one would expect to see a small branch on each T-T plot with a slope corresponding to a lower spectral index.

Compact sources in the survey total-intensity data appear to have been broadened by about $20 \%$ beyond the beamwidths shown in Figure 7. This is probably the result of the scanning strategy, where neighboring scans-needed for accurate depiction of a point source-may have been spaced by hours, days, or even months, and gain drifts occurred over those time lapses. This point has been made about surveys using scanning techniques like ours by Reich \& Reich (1988).

Figure 18 presents histograms of the fractional polarization over the area of the survey, shown in three ranges of latitude. To calculate these values, it was necessary to restore the zero level of the total-intensity maps. At $400 \mathrm{MHz}$, we added back the minimum sky brightness temperature from Haslam et al. (1982). At other frequencies, we adjusted this by a factor appropriate for $\beta=-2.5$. Errors in these numbers will not seriously affect these plots. Latitude boundaries in Figure 18 


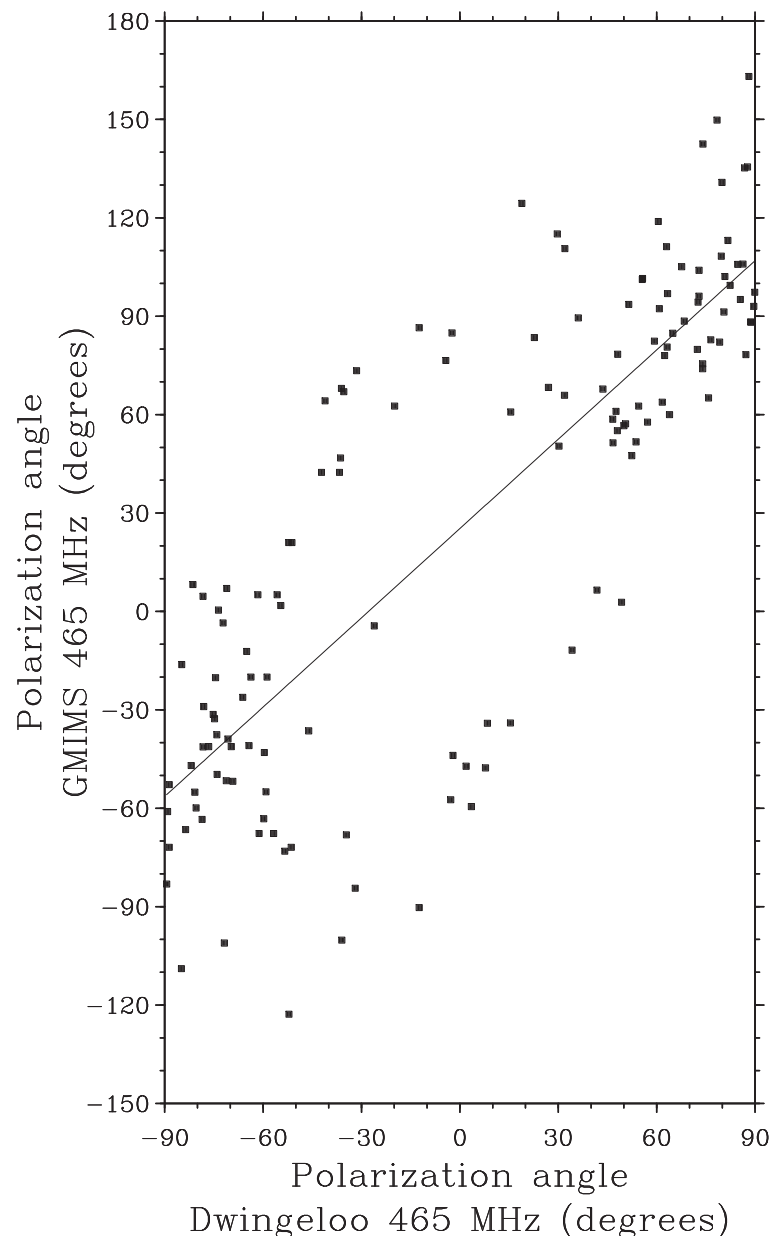

Figure 17. As for Figure 16, but for $465 \mathrm{MHz}$. The plot contains 138 points, and the slope of the fitted line is 0.91 . See text for discussion.

are chosen in steps of 0.5 in $\operatorname{cosec}|b|$. Assuming a plane-parallel magneto-ionic medium (MIM), parallel to the Galactic plane, $\operatorname{cosec}|b|$ is the ratio of the path length through the MIM to its scale height.

Three effects are evident in the data. First, fractional polarization is generally very low: depolarization is strong along most sightlines. Second, fractional polarization is slightly higher at higher frequencies. This can be understood in terms of the polarization horizon, the maximum distance from which polarized emission can be received at a particular frequency and beamwidth (Uyanıker et al. 2003). Both depth depolarization and beam depolarization determine the polarization horizon, and both are reduced at higher frequencies. Third, independent of frequency, the highest latitude range displays slightly higher fractional polarization, but only in a small number of directions. In a handful of directions, we may be "seeing" beyond the half-height of the MIM layer.

Artifacts appear in the $Q$ and $U$ images around a few strong sources (e.g., Virgo A, Hydra A, Fornax A, Pictor A). These are the product of cross-polarization in the feed and show the characteristic "four-leaf clover" pattern, alternating positive and negative lobes spaced $\frac{\pi}{2}$ around the source; the $Q$ and $U$ patterns differ by $45^{\circ}$. These lobes are at a level of a few percent. They arise from cross-polarization in the feed, converting $I$ into $Q$ and $U$. The same effect produces apparently polarized emission along the Galactic plane where total-intensity emission is very strong (see Figures 19 and 20). This too is spurious.

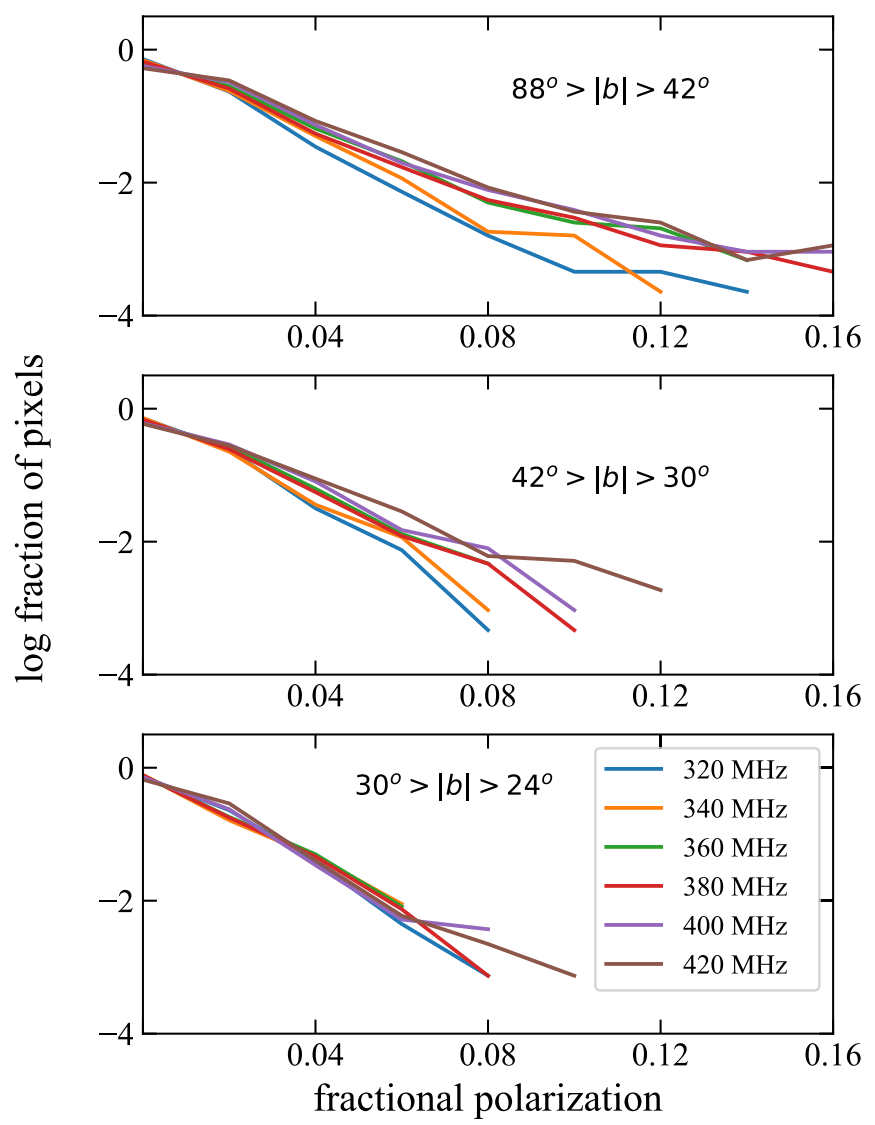

Figure 18. Histograms showing fractional polarization at frequencies across the band of the survey in three ranges of Galactic latitude. Calculation of fractional polarization followed procedures described in the text.

The unprecedented data product from this survey is the Faraday-depth cube. Dickey et al. (2019) calculate the moments of Faraday depth from this cube, from order zero to order two. Here, we present images of the zeroth and first moments. The zeroth moment (top image in Figure 19) shows the total polarized brightness integrated over the full range of Faraday depth. This is a representation of the locations of bright polarized emission without presenting maps at specific frequencies. This image bears a strong resemblance to the map of polarized intensity at $408 \mathrm{MHz}$ shown by Mathewson \& Milne (1965). The lower image of Figure 19 is the first moment map; for every pixel, it shows the weighted average value of Faraday depth in that direction. In directions where the Faraday depth spectrum is simple, this is close to a map of peak Faraday depth. Figure 20 shows images at Faraday depths of -8.5 and $+5 \mathrm{rad} \mathrm{m}^{-2}$.

Figure 21 presents a few characteristic Faraday depth spectra from the survey. We do not attempt interpretation here, but simply point out the existence of multiple Faraday-depth components at some positions, and the fact that significant emission is found at nonzero values of Faraday depth. In some of these directions, the true Faraday depth spectrum may be a single wide peak: it is possible that our survey sees two peaks because we are not sensitive to Faraday depth structures wider than $8.6 \mathrm{rad} \mathrm{m}^{-2}$ (see Table 1). Schnitzeler et al. (2009) similarly report complex Faraday spectra seen in highresolution $(2 ! 8 \times 4 ! 7)$ data covering $324-387 \mathrm{MHz}$. We note the very complicated spectrum at $\ell, b=257^{\circ} .0,13^{\circ} .0$ : this 

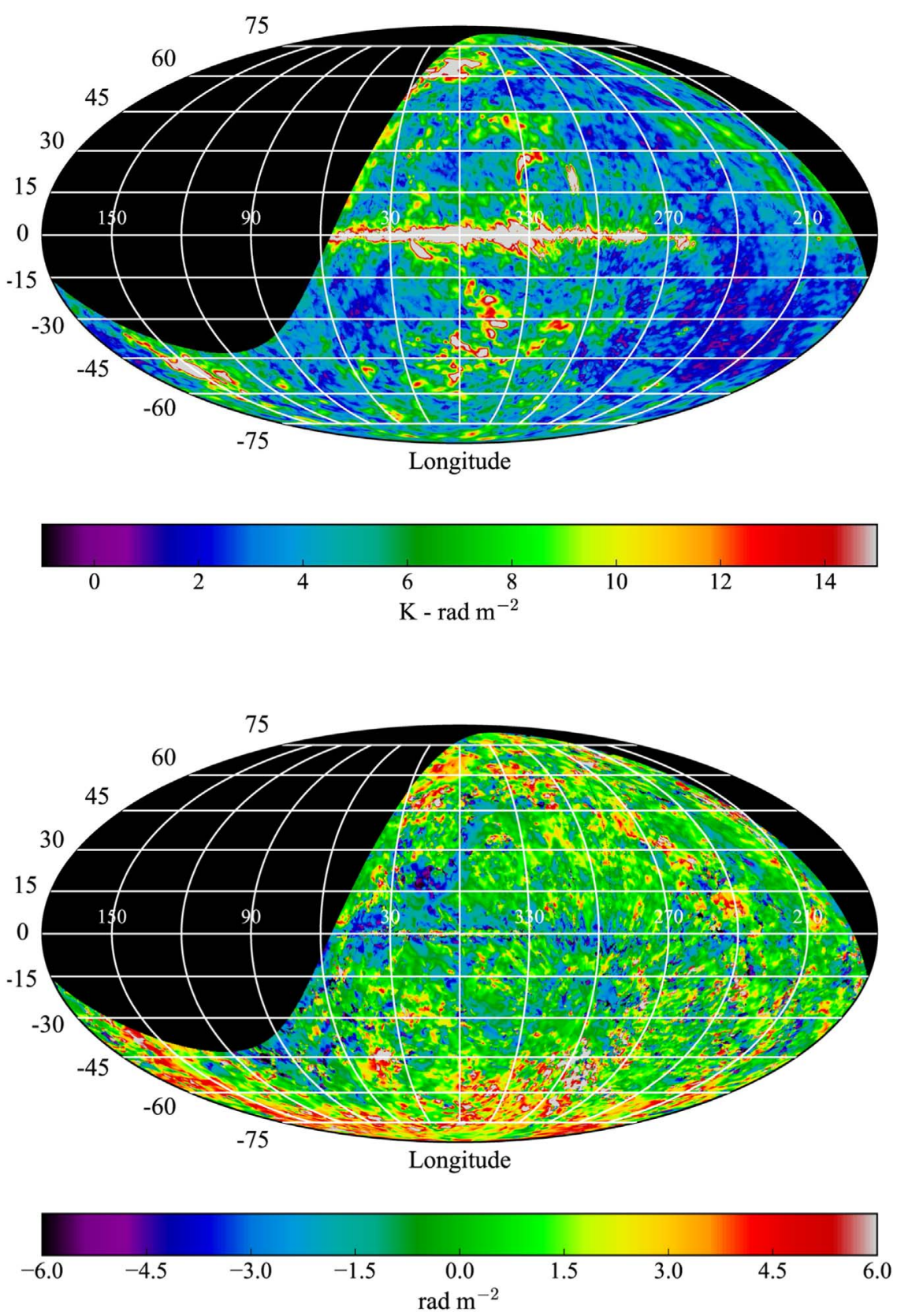

Figure 19. Zero-moment map (top) and first-moment map (bottom) calculated from the Faraday depth cube. Both are in Galactic coordinates, shown in Mollweide projection.

position is on the southern part of the Gum Nebula (Purcell et al. 2015).

Given the significant problems that we encountered with RFI, the survey data products are not noise-limited. Scanning artifacts remain in the images, and they are probably attributable to remnants of low-level RFI. Fluctuations on images are at levels of $1 \mathrm{~K} \mathrm{rms}$ in total-intensity maps and $120 \mathrm{mK}$ rms in $Q$ and $U$ maps at a beamwidth of $1^{\circ} .35$. Fluctuations on images in the Faraday depth cube are $\sim 20 \mathrm{mK}$ in single channels of width $0.5 \mathrm{rad} \mathrm{m}^{-2}$.

\section{Concluding Discussion}

We have described a large survey of polarized emission, covering $67 \%$ of the visible sky. We have demonstrated that the rapid azimuth scanning technique developed by Carretti for
S-PASS is a viable technique for making polarization observations of the linearly polarized sky at low frequencies. The intensity scale is absolutely calibrated at all frequencies, and is accurate to $7 \%$. Processing has concentrated on the extended emission; users of the data should be aware that some compact sources may not be accurately portrayed. Our choice of parameters for RM synthesis (Section 5.9) may not suit every application. We encourage users of the data to employ their own routines, or to apply improved routines that may be developed in the future.

This is the first of the GMIMS surveys to reach publication, providing all-sky coverage of the diffuse Galactic emission with the application of RM Synthesis. The resolution of the survey in Faraday depth is $5.9 \mathrm{rad} \mathrm{m}^{-2}$, and the survey is capable of capturing Faraday depth features with a width of $8.6 \mathrm{rad} \mathrm{m}^{-2}$. Our results demonstrate that there is emission at 

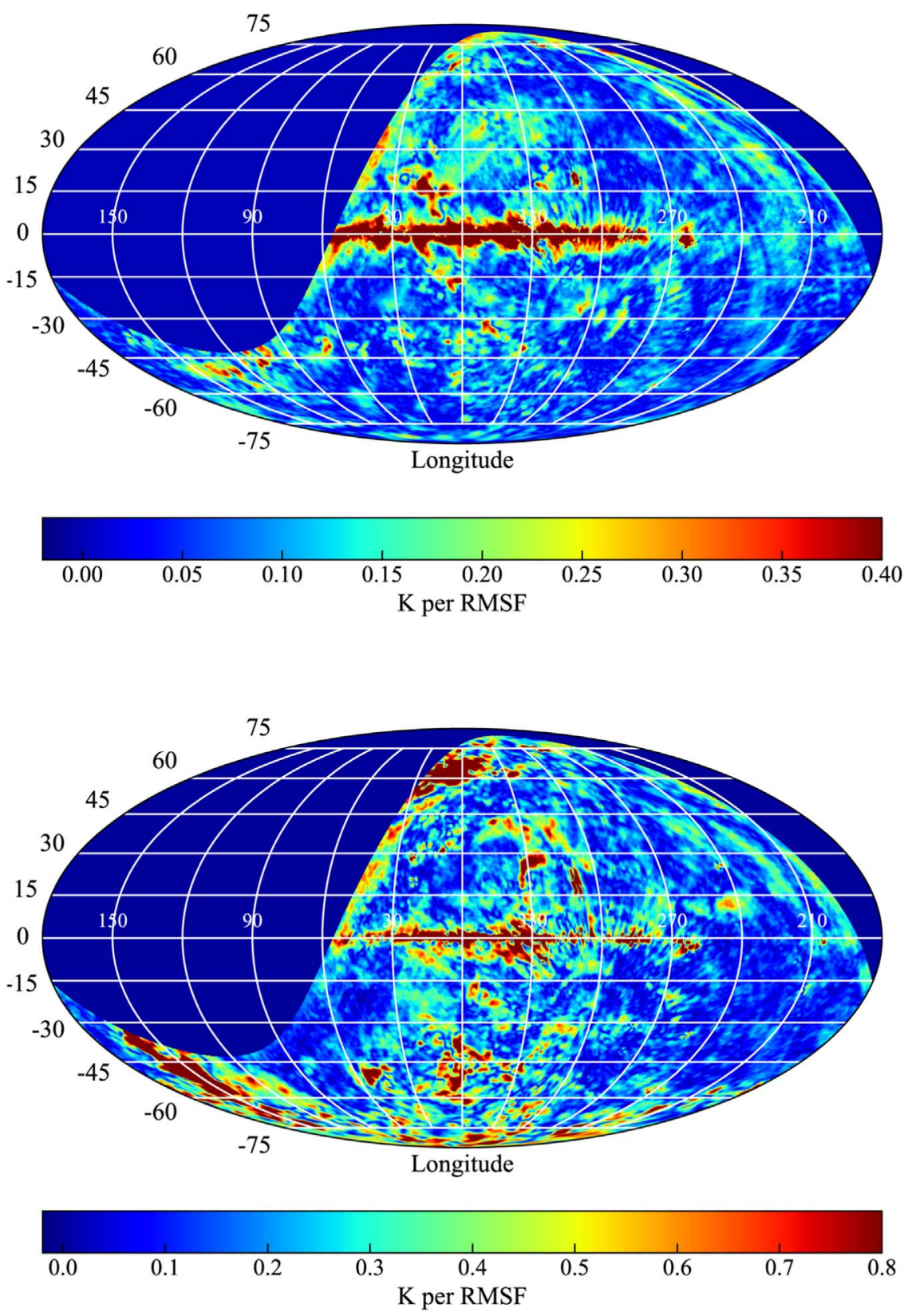

Figure 20. Faraday depth images at values of -8.5 (top) and $+5 \mathrm{rad} \mathrm{m}^{-2}$ (bottom). Note the different intensity scales for the two plots. Both are in Galactic coordinates, shown in Mollweide projection.

nonzero Faraday depths. Many directions display Faraday depth spectra with multiple peaks-commonly two, but sometimes more. There is polarized emission beyond the reach of single-frequency surveys. Had we been able to use data up to $900 \mathrm{MHz}$, we would have been able to "see" structures as wide as $\sim 30 \mathrm{rad} \mathrm{m}^{-2}$ : good resolution in Faraday depth requires observing to long wavelengths, and the ability to image broad Faraday depth features requires broad coverage in wavelength.

Wideband observations like this survey necessarily stray outside the traditional radio astronomy frequency allocationswhich are, in any event, quite narrow. From our experience, we have learned that successful observing is possible even under conditions of quite intense RFI. The key to our success was heavy spatial oversampling of the sky. Because the survey was planned for full sampling at $900 \mathrm{MHz}$, we observed every point of the $300 \mathrm{MHz}$ sky on the order of ten times. This proved to be sufficient to achieve a very high fractional coverage of the survey area. This has been achieved, of course, at the expense of valuable telescope time and observer time, but there seems to be no simple alternative. We note that this kind of oversampling can be successful as long as the RFI is intermittent, even if band occupancy is high. Nevertheless, we must acknowledge that our images are not noise-limited: they are artifact-limited, and the artifacts are probably RFI at levels below our excision process. We cut off the RFI excision at a level where we judged that the data contained valid and useful astrophysical information.

In our case, astrophysical requirements would have dictated a channel width no narrower than a few $\mathrm{MHz}$, but using narrower bandwidths meant that data affected by RFI could be deleted without losing a significant amount of good data. It is best if the channel width matches the width of the RFI signals, and our use of $0.5 \mathrm{MHz}$ channels came close to this. Current 


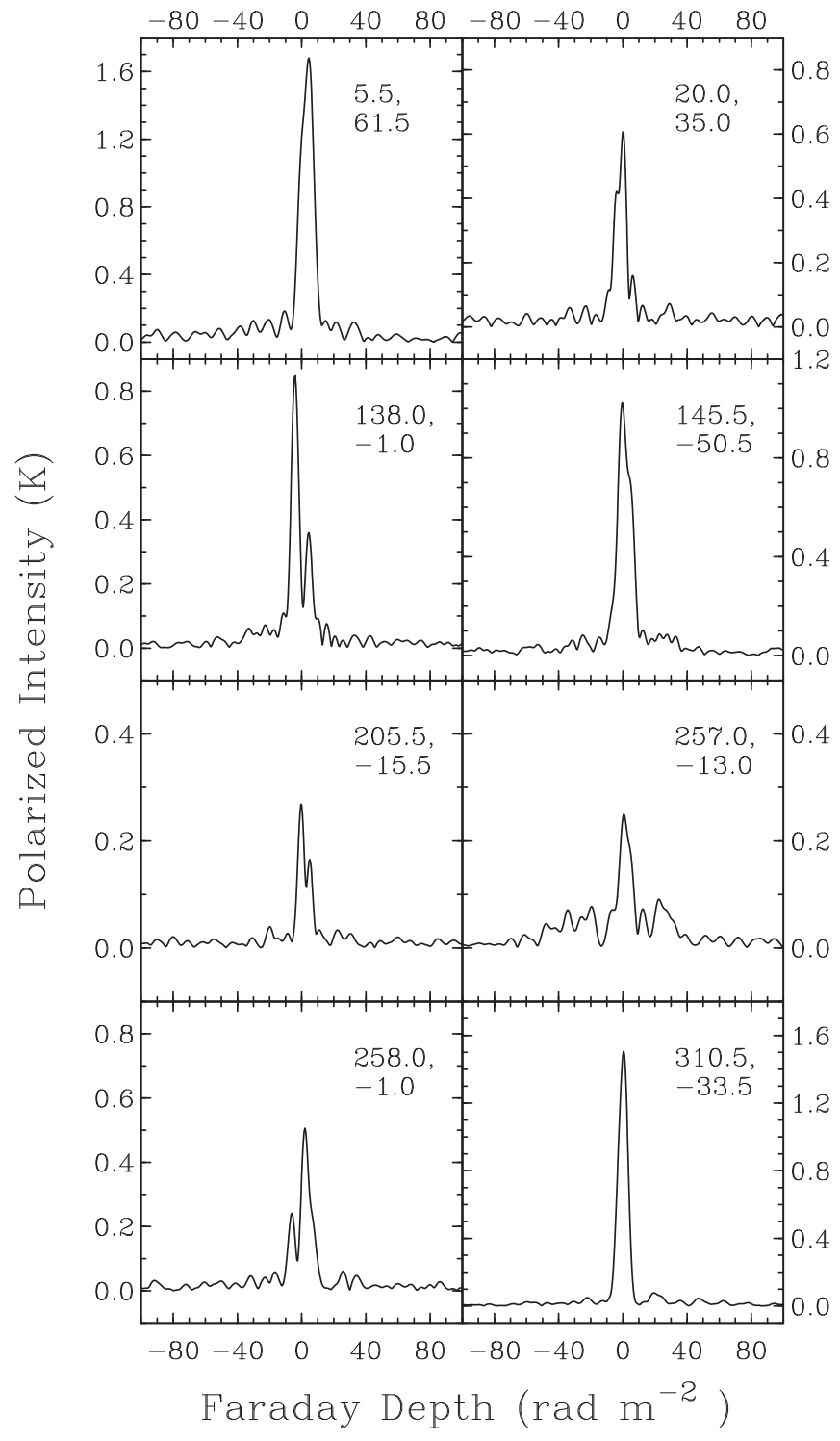

Figure 21. Faraday depth spectra. Sky position in Galactic coordinates is shown for each spectrum. Different vertical scales are used for some positions.

digital data processing systems are capable of delivering narrow channel widths, and they should be used.

Our survey is absolutely calibrated in amplitude. This was necessary because we observed well away from traditional radio astronomy bands and there were few data that we could use to transfer calibration. The fact that our data are published in units of Kelvins of brightness temperature is important to allow us to link this survey to other GMIMS data products. The intensity scale compares well at $408 \mathrm{MHz}$ with the scale of the survey of Haslam et al. (1982). Beyond that comparison, only internal checks on the amplitude scale are possible. These depend on assumptions about the spectral index of synchrotron emission, and they have limited precision because of the narrow frequency range. The weakest point of our calibration is the calibration of polarization angle, and we have ultimately been compelled to use the excellent data of Brouw \& Spoelstra (1976). Nevertheless, the most important data product from our survey is the Faraday depth cube: for many purposes, the actual polarization angles are less important than the complex change of angle as a function of frequency.
The data presented in this paper are available at the Canadian Astronomy Data Centre at doi:11570/18.0007.

Rob Messing, Richard Hellyer, and Ev Sheehan of DRAO put great skill and hard work into building the receiver with us. The Parkes Radio Telescope is part of the Australia Telescope National Facility, which is funded by the Commonwealth of Australia for operation as a national facility managed by CSIRO. We could not have started this survey without the help of the staff at the Parkes Observatory, and their endless enthusiasm helped us bring it to fruition: John Reynolds, Brett Armstrong, Ken Reeves, and Mal Smith helped us extensively with receiver and RFI issues. Mike Kesteven contributed to the development of the telescope drive software that made fast scanning possible. Jana Koehler, Stasi Baran, Niloofar Gheissari, Xiaohui Sun, LiGang Hou, and Dominic Schnitzeler helped with observations. In processing the survey data, we used the facilities of the Centre for High Performance Computing in Capetown, South Africa. Tony Willis provided valuable help with calculations of ionospheric Rotation Measure. The Max-Planck-Institut für Radioastronomie provided some essential components for the receiver used in this work. M.W. was supported in part by funds from the Natural Sciences and Engineering Research Council. A.F. thanks the STFC and the Leverhulme Trust for financial support. The Dunlap Institute is funded through an endowment established by the David Dunlap family and the University of Toronto. Sean Dougherty and Roland Kothes were generous in their support of this project from outset to completion. Finally, this paper has benefited greatly from a thorough review by a knowledgeable referee.

\section{ORCID iDs}

J. M. Dickey (iD https://orcid.org/0000-0002-6300-7459 N. M. McClure-Griffiths (i) https://orcid.org/0000-00032730-957X

\section{References}

Aller, H. D., \& Reynolds, S. P. 1985, ApJL, 293, L73

Armstrong, J. W., Rickett, B. J., \& Spangler, S. R. 1995, ApJ, 443, 209

Baars, J. W. M., Genzel, R., Pauliny-Toth, I. I. K., \& Witzel, A. 1977, A\&A, 61, 99

Baker, J. R., \& Wilkinson, A. 1974, MNRAS, 167, 581

Ben Bekhti, N., Flöer, L., Keller, R., et al. 2016, A\&A, 594, A116

Berkhuijsen, E. M., \& Brouw, W. N. 1963, BAN, 17, 185

Bilitza, D. 2015, AdSpR, 55, 1914

Bingham, R. G. 1966, MNRAS, 134, 327

Boulares, A., \& Cox, D. P. 1990, ApJ, 365, 544

Brentjens, M. A., \& de Bruyn, A. G. 2005, A\&A, 441, 1217

Brouw, W. N., \& Spoelstra, T. A. T. 1976, A\&AS, 26, 129

Brown, J. C. 2010, in ASP Conf. Ser. 438, The Dynamic Interstellar Medium:

A Celebration of the Canadian Galactic Plane Survey, ed. R. Kothes,

T. L. Landecker, \& A. G. Willis (San Francisco, CA: ASP), 216

Brown, J. C., Haverkorn, M., Gaensler, B. M., et al. 2007, ApJ, 663, 258

Burn, B. J. 1966, MNRAS, 133, 67

Carretti, E., Haverkorn, M., Staveley-Smith, L., et al. 2019, MNRAS, in press (arXiv:1903.09420)

Chepurnov, A., \& Lazarian, A. 2010, ApJ, 710, 853

Cooper, B. F. C., \& Price, R. M. 1962, Natur, 196, 761

Costain, C. H. 1960, MNRAS, 120, 248

CST 2014, CST Microwave Studio, Workflow and Solver Overview (Darmstadt: Computer Simulation Technology AG)

de Avillez, M. A., \& Breitschwerdt, D. 2004, Ap\&SS, 289, 479

de Avillez, M. A., \& Breitschwerdt, D. 2005, A\&A, 436, 585

de Bruyn, A. G., \& Brentjens, M. A. 2005, A\&A, 441, 931

Dickey, J. M., Landecker, T. L., Thomson, A. J. M., et al. 2019, ApJ, 871, 106 
Dickey, J. M., McClure-Griffiths, N. M., Gibson, S. J., et al. 2013, PASA, 30, 3 Du, X., Landecker, T. L., Robishaw, T., et al. 2016, PASP, 128, 115006 Ferrière, K. M. 2001, RvMP, 73, 1031

Gardner, F. F., \& Whiteoak, J. B. 1963, Natur, 197, 1162

Gressel, O., Elstner, D., Ziegler, U., \& Rüdiger, G. 2008, A\&A, 486, L35

Haffner, L. M., Reynolds, R. J., Madsen, G. J., et al. 2010, in ASP Conf. Ser. 438, The Dynamic Interstellar Medium: A Celebration of the Canadian Galactic Plane Survey, ed. R. Kothes, T. L. Landecker, \& A. G. Willis (San Francisco, CA: ASP), 388

Haffner, L. M., Reynolds, R. J., Tufte, S. L., et al. 2003, ApJS, 149, 405

Han, J. L. 2017, ARA\&A, 55, 111

Han, J. L., Manchester, R. N., Lyne, A. G., Qiao, G. J., \& van Straten, W. 2006, ApJ, 642, 868

Haslam, C. G. T., Klein, U., Salter, C. J., et al. 1981, A\&A, 100, 209

Haslam, C. G. T., Salter, C. J., Stoffel, H., \& Wilson, W. E. 1982, A\&AS, 47, 1

Haverkorn, M., Gaensler, B. M., McClure-Griffiths, N. M., Dickey, J. M., \& Green, A. J. 2006, ApJS, 167, 230

Heald, G. 2009, in IAU Symp. 259, Cosmic Magnetic Fields: From Planets, to Stars and Galaxies, ed. K. G. Strassmeier, A. G. Kosovichev, \& J. E. Beckman (Cambridge: Cambridge Univ. Press), 591

Heiles, C., \& Haverkorn, M. 2012, SSRv, 166, 293

Helmboldt, J. F., Kassim, N. E., Cohen, A. S., Lane, W. M., \& Lazio, T. J. 2008, ApJS, 174, 313

Hill, A. S., Joung, M. R., Mac Low, M.-M., et al. 2012, ApJ, 750, 104

Hill, A. S., Landecker, T. L., Carretti, E., et al. 2017, MNRAS, 467, 4631

Jansson, R., \& Farrar, G. R. 2012, ApJ, 757, 14

Joung, M. R., Mac Low, M.-M., \& Bryan, G. L. 2009, ApJ, 704, 137

Kalberla, P. M. W., Burton, W. B., Hartmann, D., et al. 2005, A\&A, 440, 775

Kerp, J., Winkel, B., Ben Bekhti, N., Flöer, L., \& Kalberla, P. M. W. 2011, AN, 332, 637

Kildal, P.-S. 2005, Broadband Multi-Dipole Antenna with FrequencyIndependent Radiation Characteristic, International Patent WO 2005/ 015685 A1 https://patents.google.com/patent/US8130162B2/en

Landecker, T. L. 1969, PhD thesis, Univ. Sydney

Landecker, T. L., Reich, W., Reid, R. I., et al. 2010, A\&A, 520, A80

Li, M., Ostriker, J. P., Cen, R., Bryan, G. L., \& Naab, T. 2015, ApJ, 814, 4

Liao, Y.-W., Chang, T.-C., Kuo, C.-Y., et al. 2016, ApJ, 833, 289

Mathewson, D. S., Broten, N. W., \& Cole, D. J. 1966, AuJPh, 19, 93

Mathewson, D. S., \& Milne, D. K. 1965, AuJPh, 18, 635

McClure-Griffiths, N. M., Dickey, J. M., Gaensler, B. M., et al. 2005, ApJS, 158,178

McClure-Griffiths, N. M., Dickey, J. M., Gaensler, B. M., et al. 2012, ApJS, 199, 12

McClure-Griffiths, N. M., Pisano, D. J., Calabretta, M. R., et al. 2009, ApJS, 181,398
McKee, C. F., \& Ostriker, J. P. 1977, ApJ, 218, 148

Mevius, M. 2018, RMextract: Ionospheric Faraday Rotation Calculator, Astrophysics Source Code Library, ascl:1806.024

Minter, A. H., \& Spangler, S. R. 1996, ApJ, 458, 194

Olsson, R., Kildal, P.-S., \& Weinreb, S. 2006, ITAP, 54, 368

Ostriker, E. C., McKee, C. F., \& Leroy, A. K. 2010, ApJ, 721, 975

Pauliny-Toth, I. K., \& Shakeshaft, J. R. 1962, MNRAS, 124, 61

Peek, J. E. G., Babler, B. L., Zheng, Y., et al. 2018, ApJS, 234, 2

Peek, J. E. G., Heiles, C., Douglas, K. A., et al. 2011, ApJS, 194, 20

Piontek, R. A., \& Ostriker, E. C. 2007, ApJ, 663, 183

Planck Collaboration, Adam, R., Ade, P. A. R., et al. 2016, A\&A, 596, A103

Puglia, K. V. 2002, IMMag, 3, 56

Purcell, C. R., Gaensler, B. M., Sun, X. H., et al. 2015, ApJ, 804, 22

Reich, P., \& Reich, W. 1988, A\&AS, 74, 7

Schnitzeler, D. H. F. M., Katgert, P., \& de Bruyn, A. G. 2009, A\&A, 494, 611

Seymour, P. A. H. 1966, MNRAS, 134, 389

Simard-Normandin, M., \& Kronberg, P. P. 1979, Natur, 279, 115

Sironi, G. 1974, MNRAS, 166, 345

Slavin, J. D., \& Cox, D. P. 1993, ApJ, 417, 187

Spoelstra, T. A. T. 1984 , A\&A, 135, 238

Sun, X. H., Landecker, T. L., Gaensler, B. M., et al. 2015a, ApJ, 811, 40

Sun, X. H., Reich, W., Waelkens, A., \& Enßlin, T. A. 2008, A\&A, 477, 573

Sun, X. H., Rudnick, L., Akahori, T., et al. 2015b, AJ, 149, 60

Tapping, K. F. 2013, SpWea, 11, 394

Taylor, A. R., Gibson, S. J., Peracaula, M., et al. 2003, AJ, 125, 3145

Taylor, A. R., Stil, J. M., \& Sunstrum, C. 2009, ApJ, 702, 1230

Testori, J. C., Reich, P., \& Reich, W. 2008, A\&A, 484, 733

Thébault, E., Finlay, C. C., Beggan, C. D., et al. 2015, EP\&S, 67, 79

Uyanıker, B., Landecker, T. L., Gray, A. D., \& Kothes, R. 2003, ApJ, 585, 785

Vinyaikin, E. N. 2007, ARep, 51, 570

Webster, A. S. 1974, MNRAS, 166, 355

Westerhout, G., Seeger, C. L., Brouw, W. N., \& Tinbergen, J. 1962, BAN, 16, 187

Wheeler, H. A. 1959, Proc. IRE, 47, 1325

Wielebinski, R., Shakeshaft, J. R., \& Pauliny-Toth, I. I. K. 1962, Obs, 82, 158

Wilkinson, A. 1973, MNRAS, 163, 147

Wolfire, M. G., McKee, C. F., Hollenbach, D. J., \& Tielens, A. G. G. M. 2003, ApJ, 587, 278

Wolleben, M., Fletcher, A., Landecker, T. L., et al. 2010, ApJL, 724, L48

Wolleben, M., Landecker, T. L., Carretti, E., et al. 2009, in IAU Symp. 259, Cosmic Magnetic Fields: From Planets, to Stars and Galaxies, ed. K. G. Strassmeier, A. G. Kosovichev, \& J. E. Beckman (Cambridge: Cambridge Univ. Press), 89

Wolleben, M., Landecker, T. L., Reich, W., \& Wielebinski, R. 2006, A\&A, 448, 411

Yang, J., Pantaleev, M., Kildal, P.-S., et al. 2011, ITAP, 59, 1918 